\title{
RELATIVE ENTROPIES \\ FOR KINETIC EQUATIONS IN BOUNDED DOMAINS \\ (irreversibility, stationary solutions, uniqueness)
}

\author{
Naoufel BEN ABDALLAH ${ }^{1}$, Jean DOLBEAULT ${ }^{2}$ \\ 1 M.I.P. (UMR CNRS no. 5640), \\ Université Paul Sabatier - Toulouse III, \\ 118, route de Narbonne, 31062 Toulouse Cedex 3, France. \\ E-mail: naoufel@mip.ups-tlse.fr \\ ${ }^{2}$ Ceremade (UMR CNRS no. 7534), \\ Université Paris IX - Dauphine, \\ Place de Lattre de Tassigny, 75775 Paris Cedex 16, France. \\ E-mail: dolbeaul@ceremade.dauphine.fr
}

\begin{abstract}
The relative entropy method describes the irreversibility of the Vlasov-Poisson and VlasovBoltzmann-Poisson systems in bounded domains with incoming boundary conditions. Uniform in time estimates are deduced from the entropy. In some cases, these estimates are sufficient to prove the convergence of the solution to a unique stationary solution, as time goes to infinity. The method is also used to analyze other types of boundary conditions such as mass and energy preserving diffuse reflection boundary conditions, and to prove the uniqueness of stationary solutions for some special collision terms.
\end{abstract}

Keywords. Kinetic equations - Vlasov-Poisson system - Boltzmann equation - collision kernels irreversibility - $H$-Theorem - injection boundary conditions - diffusive boundary conditions - relative entropy - large time asymptotics - uniqueness - stationary solutions - minimization under constraints - nonlinear stability - Casimir energy - Bolza problem - plasmas - semi-conductors.

AMS classification (2000). 35B30, 82C40. Secondary: 35B40, 35B45, 35A05, 82D05, 82D10, 82D 37, 82B40.

\section{$1 \quad$ Preliminaries and examples}

In this paper, we study the large time behaviour of the solutions of the initial-boundary value problem for the Vlasov-Poisson and the Vlasov-Poisson-Boltzmann systems. We consider charged particles (say electrons) described by their distribution function $f(x, v, t)$ where $x$ is the position of the particle, $v$ its velocity and $t$ is the time variable. The position $x$ is assumed to lie in a bounded domain $\omega$ of $\mathbb{R}^{d}(d=1,2,3)$. The velocity variable $v$ is in $\mathbb{R}^{d}$ whereas the time variable $t$ is in $\mathbb{R}^{+}$. We shall assume that the particles are submitted to an electric field $E=E(x, t)$ deriving from the sum of an external potential $\phi_{0}(x)$ and a self-consistent potential $\phi(x, t)$ created by the electrons themselves through the Coulomb interaction. The potential $\phi_{0}(x)$ is a given stationary function which can be seen for instance as the result of an applied voltage and a background ion density, whereas $\phi$ is a solution of the Poisson equation with homogeneous Dirichlet boundary conditions on $\partial \omega$. We shall also assume that electrons may suffer collisions modelled by a collision operator $Q(f)$. We consider the Cauchy problem corresponding to an initial distribution function $f_{0}$ at time $t=0$. Various boundary 
conditions for $f$ will be presented. We shall however detail the case where the distribution function of incoming particles is prescribed.

Notations. We recall that the spatial domain is denoted by $\omega$. From now on, we assume that $\omega$ is bounded and $\partial \omega$ is of class $C^{1}$. We shall denote by $\Omega=\omega \times \mathbb{R}^{d}$ and $\Gamma=\partial \Omega=\partial \omega \times \mathbb{R}^{d}$ the phase space and its boundary respectively. Let $d_{\partial \omega}$ be the surface measure induced on $\partial \omega$ by Lebesgue's measure. The outward unit normal vector at a point $x$ of $\partial \omega$ is denoted by $\nu(x)$. For any given $x \in \partial \omega$, we set

$$
\Sigma^{ \pm}(x)=\left\{v \in \mathbb{R}^{d}: \pm v \cdot \nu(x)>0\right\} \quad \text { and } \quad \Gamma^{ \pm}=\left\{(x, v) \in \Gamma: v \in \Sigma^{ \pm}(x)\right\} .
$$

Finally, $d \sigma(x, v)$ stands for the measure $|\nu(x) \cdot v| d_{\Gamma}(x, v)$ where $d_{\Gamma}(x, v)=d_{\partial \omega}(x) d v$ is the measure induced by Lebesgue's measure on $\Gamma$. By a standard abuse of notations, we will not distinguish a function and its trace on the boundary.

On $\Gamma^{-}$, we shall assume that the distribution function $f(x, v, t)$ is a given function of the incoming velocities, which actually only depends on the total energy $|v|^{2} / 2+\phi_{0}(x)$. On $\Omega$, the distribution function $f$ and the electrostatic potential $\phi$ are a solution of the Vlasov-Poisson-Boltzmann system. The initial-boundary problem for $f$ and $\phi$ can therefore be written as

$$
\left\{\begin{aligned}
& \partial_{t} f+v \cdot \nabla_{x} f-\left(\nabla_{x} \phi+\nabla_{x} \phi_{0}\right) \cdot \nabla_{v} f=Q(f), \quad(x, v) \in \Omega, t \in \mathbb{R}^{+}, \\
& \text {and } \quad f_{\mid t=0}=f_{0}, f_{\mid \Gamma^{-} \times \mathbb{R}^{+}}(x, v, t)=\gamma\left(\frac{1}{2}|v|^{2}+\phi_{0}(x)\right), \\
&-\Delta \phi=\rho=\int_{\mathbb{R}^{d}} f d v,(x, t) \in \omega \times \mathbb{R}^{+}, \\
& \text {and } \phi(x, t)=0,(x, t) \in \partial \omega \times \mathbb{R}^{+} .
\end{aligned}\right.
$$

\subsection{Assumptions}

Throughout this paper, we shall use the 5 following assumptions:

(H1) The initial condition $f_{0}$ is a nonnegative function with integrability properties and $L^{\infty}$ bounds that will be specified later on.

(H2) The external electrostatic potential is assumed to be in $C^{2}(\bar{\omega})$. Without loss of generality we assume that $\phi_{0} \geq 0$.

(H3) The function $\gamma$ has the following property

\section{Property $\mathcal{P}$}

The function $\gamma$ is defined on $\left(\min _{x \in \omega} \phi_{0}(x),+\infty\right)$, bounded, smooth, strictly decreasing with values in $\mathbb{R}_{*}^{+}$, and rapidly decreasing at infinity, so that

$$
\sup _{x \in \omega} \int_{0}^{+\infty} s^{d / 2} \gamma\left(s+\phi_{0}(x)\right) d s<+\infty .
$$

We denote by $\gamma^{-1}$ its inverse function to $\mathbb{R}$ extended by an arbitrary, fixed, strictly decreasing function.

(H4) The collision operator $Q$ is assumed to preserve the mass $\int_{\mathbb{R}^{d}} Q(g) d v=0$, and satisfies the following $H$-theorem

$$
D[g]=-\int_{\mathbb{R}^{d}} Q(g)\left[\frac{1}{2}|v|^{2}-\gamma^{-1}(g)\right] d v \geq 0,
$$

for any nonnegative function $g$ in $L^{1}\left(\mathbb{R}^{d}\right)$.

(H5) We assume that

$$
D[g]=0 \Longleftrightarrow Q(g)=0 .
$$


The aim of this paper is to study the irreversibility of the system (1), the uniqueness of the stationary solutions and the eventual convergence to a stationary solution for large time asymptotics. The main ingredient is the derivation of a $\gamma$-dependent relative entropy of the time-dependent solution versus a stationary solution of the problem. In order to exhibit such a stationary solution, we introduce the map $U$, defined on $L^{1}(\Omega)$ in the following way: for any function $g \in L^{1}(\Omega)$, we denote by $U[g]=u$ the unique solution in $W_{0}^{1, d /(d-1)}(\omega)$ of

$$
-\Delta u=\int_{\mathbb{R}^{d}} g(x, v) d v .
$$

The operator $U$ is linear and satisfies

$$
\int_{\Omega} g U[f] d x d v=\int_{\Omega} f U[g] d x d v \quad \text { and } \quad \int_{\Omega} f U[f] d x d v=\int_{\omega}\left|\nabla_{x} U[f]\right|^{2} d x .
$$

Moreover, it is readily seen in view of Hypotheses (H4)-(H5) on $Q$, that any function $M$ satisfying

$$
M(x, v)=\gamma\left(\frac{1}{2}|v|^{2}+U[M](x)+\phi_{0}(x)\right) \quad \forall(x, v) \in \Omega
$$

is a stationary solution of $(1)$, satisfying $Q(M)=0$. Indeed, letting $\phi(x)=U[M]$, we obviously have

$$
v \cdot \nabla_{x} M-\left(\nabla_{x} \phi_{0}+\nabla_{x} \phi\right) \cdot \nabla_{v} M=0 \quad \text { with } \quad-\Delta \phi=\int_{\mathbb{R}^{d}} M d v .
$$

Finally, we deduce from the mass conservation that

$$
\int_{\mathbb{R}^{d}} Q(M)\left(\frac{1}{2}|v|^{2}-\gamma^{-1}(M)\right) d v=-\int_{\mathbb{R}^{d}} Q(M)\left(\phi(x)+\phi_{0}(x)\right) d v=0,
$$

which leads to $Q(M)=0$ thanks to (3). Boundary conditions are obviously satisfied by $M$ and $U[M]$. It is not difficult to prove that $M$ defined by (4) exists and is actually unique since $U[M]$ is the unique critical point in $H_{0}^{1}(\omega)$ of the strictly convex coercive functional

$$
U \mapsto \frac{1}{2} \int_{\omega}|\nabla U|^{2} d x-\int_{\omega} G\left(U+\phi_{0}\right) d x,
$$

where $G$ is a primitive of $g(u)=\int_{\mathbb{R}^{d}} \gamma\left(\frac{1}{2}|v|^{2}+u\right) d v=2^{d / 2-1}\left|S^{d-1}\right| \cdot \int_{0}^{+\infty} s^{d / 2-1} \gamma(s+u) d s$.

\subsection{Examples}

In this paragraph, we list typical examples in which we are interested and show that Assumptions (H1)(H5) are satisfied by wide classes of mutually compatible collision operators and boundary conditions.

Example 1 : Pure Vlasov-Poisson system. The collision operator $Q$ is identically vanishing. The inflow function $\gamma$ is any arbitrary decreasing function satisfying Property $(\mathcal{P})$.

Example 2 : The Vlasov-Poisson-Fokker-Planck system. The collision operator $Q_{F P}$ is defined by

$$
Q_{F P}(f)=\operatorname{div}_{v}\left(v f+\theta \nabla_{v} f\right)
$$

where $\theta>0$ is a given constant temperature. A slightly more general form of this operator is

$$
Q_{F P, \alpha}(f)=\operatorname{div}_{v}\left(v f(1-\alpha f)+\theta \nabla_{v} f\right)
$$

where $\alpha$ is a nonnegative constant. The case $\alpha>0$ corresponds to a nonlinear diffusive Fokker-Planck operator for which the Pauli exclusion principle is taken into account. This operator satisfies the following $H$-theorem

$$
\int_{\mathbb{R}^{d}} Q_{F P, \alpha}(f) \log \left(\frac{f}{(1-\alpha f) M_{\theta}}\right) d v=-\int_{\mathbb{R}^{d}} \theta f(1-\alpha f)\left|\nabla_{v} \log \left(\frac{f}{(1-\alpha f) M_{\theta}}\right)\right|^{2} d v
$$

where $M_{\theta}=(2 \pi \theta)^{-d / 2} e^{-|v|^{2} /(2 \theta)}$. 
Lemma 1.1 Let $\alpha \geq 0$ and consider the set of admissible functions for $Q_{F P, \alpha}$ defined by

$$
A_{\alpha}^{1}=\left\{f \in L^{1}\left(\mathbb{R}_{v}^{d}\right): 0 \leq f \leq \alpha^{-1} \text { a.e. and } \quad v \sqrt{f(1-\alpha f)}, \nabla \sqrt{f(1-\alpha f)} \in L^{2}\left(\mathbb{R}_{v}^{d}\right)\right\}
$$

where we take the convention: $\alpha^{-1}=+\infty$ if $\alpha=0$. Then for any $f \in A_{\alpha}^{1}$,

$$
\mathcal{H}(f)=\int_{\mathbb{R}^{d}} Q_{F P, \alpha}(f) \log \left(\frac{f}{(1-\alpha f) M_{\theta}}\right) d v \leq 0
$$

and the three following statements are equivalent :

1. $f \in A_{\alpha}^{1}$ and $\mathcal{H}(f)=0$.

2. $f \in A_{\alpha}^{1}$ and $Q_{F P, \alpha(f)}=0$.

3. There exists $\mu \in \mathbb{R}$ such that $f(v)=\left(\alpha+e^{\left(|v|^{2} / 2-\mu\right) / \theta}\right)^{-1}$.

It is easy to check that $\frac{f}{1-\alpha f}$ is proportional to $M_{\theta}$ if and only if $Q(f)=0$. In order to satisfy Assumption (H5), the function $\gamma$ has to be given by $\gamma(u)=\left(\alpha+e^{(u-\mu) / \theta}\right)^{-1}$, or equivalently has to satisfy $\gamma^{-1}(f)=\mu-\theta \log \left(\frac{f}{1-\alpha f}\right)$. See $[26,19]$ for related results.

Example 3 : BGK approximation of the Boltzmann operator. Consider the collision operator for fermions

$$
Q_{\alpha}(f)=\int_{\mathbb{R}^{d}} \sigma\left(v, v^{\prime}\right)\left[M_{\theta}(v) f\left(v^{\prime}\right)(1-\alpha f(v))-M_{\theta}\left(v^{\prime}\right) f(v)\left(1-\alpha f\left(v^{\prime}\right)\right)\right] d v^{\prime} .
$$

We assume that the cross-section $\sigma$ is nonnegative symmetric and $M_{\theta}(v)=(2 \pi \theta)^{-d / 2} e^{-|v|^{2} /(2 \theta)}$ is a fixed Maxwellian function with a given temperature $\theta>0$. The parameter $\alpha$ is nonnegative. We shall distinguish the linear case $\alpha=0$ and the nonlinear case $\alpha>0$. The $H$-theorem for $Q_{\alpha}$ goes as follows.

Lemma 1.2 Assume that the cross-section $\sigma$ is symmetric, positive and bounded. Let $A_{\alpha}^{2}=\{f \in$ $L^{1}\left(\mathbb{R}_{v}^{d}\right)$ : $0 \leq f \leq \alpha^{-1}$ a.e. $\}$ be the set of admissible functions for $Q_{\alpha}$. Then for any $f \in A_{\alpha}^{2}$, $Q_{\alpha}(f)$ is bounded in $L^{1}\left(\mathbb{R}_{v}^{d}\right)$ and there exists a positive constant $C$ such that $\left\|Q_{\alpha}(f)\right\|_{L^{1}} \leq C\|f\|_{L^{1}}$. Moreover, for any $f \in A_{\alpha}^{2}$, the following inequality holds

$$
\mathcal{H}(f)=\int Q_{\alpha}(f) \log \left(\frac{f}{(1-\alpha f) M_{\theta}}\right) \leq 0
$$

and the three following statements are equivalent:

1. $f \in A_{\alpha}^{2}$ and $\mathcal{H}(f)=0$.

2. $f \in A_{\alpha}^{2}$ and $Q_{\alpha}(f)=0$.

3. There exists $\mu \in \mathbb{R}$ such that $f(v)=\left(\alpha+e^{\left(|v|^{2} / 2-\mu\right) / \theta}\right)^{-1}$.

In order to satisfy Assumption (H5), the function $\gamma$ has to be chosen equal to $\gamma(u)=\left(\alpha+e^{(u-\mu) / \theta}\right)^{-1}$ for some $\mu \in \mathbb{R}$. Therefore, we have $\gamma^{-1}(f)=\mu-\theta \log \left(\frac{f}{1-\alpha f}\right)$ and Hypotheses (H4)-(H5) are nothing but the $H$-theorem (Statement 3 of Lemma 1.2).

Example 4: Linear elastic collision operator. It takes the form

$$
Q_{E}(f)=\int_{\mathbb{R}^{d}} \chi\left(v, v^{\prime}\right)\left(f\left(v^{\prime}\right)-f(v)\right) \delta\left(\left|v^{\prime}\right|^{2}-|v|^{2}\right) d v^{\prime},
$$

where $\chi$ is a symmetric positive cross-section. Let $\lambda(v)=\int_{\mathbb{R}^{d}} \chi\left(v, v^{\prime}\right) \delta\left(|v|^{2}-\left|v^{\prime}\right|^{2}\right) d v^{\prime}$. The operator $Q_{E}$ satisfies the following properties 
Lemma 1.3 Assume that $\lambda \in L^{\infty}$ and $\chi>0$ a.e. Then the operator $Q_{E}$ is bounded on $L^{1} \cap L^{\infty}\left(\mathbb{R}^{d}\right)$. Moreover, for any measurable function $\psi$ and for any increasing function $H$ on $\mathbb{R}$, we have

$$
\int_{\mathbb{R}^{d}} Q_{E}(f) \cdot \psi\left(|v|^{2}\right) d v=0 \quad \text { and } \quad \mathcal{H}(f)=\int_{\mathbb{R}^{d}} Q_{E}(f) \cdot H(f) d v \leq 0 .
$$

Finally, if $H$ is strictly increasing, the three following assertions are equivalent:

1. $\mathcal{H}(f)=0$.

2. $Q_{E}(f)=0$.

3. There exists $\psi$ such that $f(v)=\psi\left(|v|^{2}\right)$.

Consequently, any function $\gamma$ having Property $(\mathcal{P})$ satisfies Assumptions (H1)-(H5). We shall see in Section 3 that the condition $\chi>0$ a.e. can be slightly weakened.

Example 5 : Electron-Electron collision operator. The Boltzmann collision operator $Q_{e e, \alpha}^{B}$, possibly including the Pauli exclusion term (case $\alpha>0$ ) or the Fokker-Planck-Landau collision operator $Q_{e e, \alpha}^{L}$, namely

$$
\begin{aligned}
& Q_{e e, \alpha}^{B}(f)=\int_{S^{d-1} \times \mathbb{R}^{d}} B\left(\left|v-v_{*}\right|,\left(v-v_{*}\right) \cdot \omega\right)\left[f^{\prime} f_{*}^{\prime}(1-\alpha f)\left(1-\alpha f_{*}\right)-f f_{*}\left(1-\alpha f^{\prime}\right)\left(1-\alpha f_{*}^{\prime}\right)\right] d \omega d v_{*} \\
& Q_{e e, \alpha}^{L}(f)=\sum_{i, j=1}^{3} \frac{\partial}{\partial v_{i}} \int_{\mathbb{R}^{3}} B\left(\left|v-v_{*}\right|\right) \Pi_{i j}\left(v-v_{*}\right)\left(\left(1-\alpha f_{*}\right) f_{*} \frac{\partial f}{\partial v_{j}}-f(1-\alpha f) \frac{\partial f_{*}}{\partial v_{* j}}\right) d v_{*}
\end{aligned}
$$

where $\Pi_{i j}(z)=\delta_{i j}-\frac{z_{i} z_{j}}{|z|^{2}}$ respectively, with $f^{\prime}=f\left(x, v^{\prime}, t\right), f_{*}^{\prime}=f\left(x, v_{*}^{\prime}, t\right), f_{*}=f\left(x, v_{*}, t\right)$ and $\left.v^{\prime}=v-\left(\left(v-v_{*}\right) \cdot \omega\right)\right) \omega, v_{*}^{\prime}=v_{*}+\left(\left(v-v_{*}\right) \cdot \omega \omega\right.$. The operator $Q_{e e, \alpha}^{L}$ has been derived by Lemou [70] trough a grazing limit of the Boltzmann operator. The properties of these operators are summarized in the following

Lemma 1.4 With $Q_{e e, \alpha}=Q_{e e, \alpha}^{B}$ or $Q_{e e, \alpha}^{L}$, for any nonnegative function $f \in L^{1} \cap L^{\infty}\left(\mathbb{R}^{d}\right)$ such that $0 \leq f \leq \alpha^{-1}$ a.e., which decays fast enough at infinity, we have

$$
\int_{\mathbb{R}^{d}} Q_{e e, \alpha}(f)\left(\begin{array}{c}
1 \\
v \\
|v|^{2}
\end{array}\right) d v=0 \quad \text { and } \quad \mathcal{H}(f)=\int_{\mathbb{R}^{d}} Q_{e e, \alpha}(f) \log \left(\frac{f}{1-\alpha f}\right) d v \leq 0 .
$$

Moreover, if $B$ is positive, the three following assertions are equivalent:

1. $\mathcal{H}(f)=0$.

2. $Q_{e e, \alpha}(f)=0$.

3. There exist $\theta \in \mathbb{R}^{+}, v_{0} \in \mathbb{R}^{d}$ and $\mu \in \mathbb{R}$ such that $f(v)=\left(\alpha+e^{\left(\left|v-v_{0}\right|^{2} / 2-\mu\right) / \theta}\right)^{-1}$.

A compatible inflow function $\gamma$ takes the form $\gamma(u)=\left(\alpha+e^{(u-\mu) / \theta}\right)^{-1}$.

In each of the above examples, for simplicity, the velocities are taken in $\mathbb{R}^{d}$, but we could as well consider a setting for which $v, d v$ and $\frac{1}{2}|v|^{2}$ would be replaced by $\nabla_{k} \epsilon(k), d k$ and $\epsilon(k)$ respectively, thus covering the relativistic case or the periodic framework (particles in a crystal).

Also notice that we will speak generically of the Vlasov-Poisson-Boltzmann system each time there is a non-zero collision operator, although the qualification Boltzmann should be used only for the case of Example 5, with $\alpha=0$. 


\subsection{Outline of the paper and references}

We first deal with the irreversibility due to the boundary conditions and, eventually, the collision kernel (Theorem 2.1). In the one-dimensional case and under technical regularity assumptions, the large time limit solution of the Vlasov-Poisson system, which is overdetermined on the boundary, is then characterized as the unique stationary solution (Theorem 2.5). For several models with various collision kernels corresponding to the above examples, the stationary solution is also identified as the unique limit for large times of the Cauchy problem (Corollary 2.4). Without self-consistent potential, a uniqueness result (Theorem 2.6) allows to identify the asymptotic solution (Theorem 2.7) in a special case corresponding to boundary conditions which are not compatible with the collision kernel.

Irreversibility driven by collisions is a well known topic [30]. On the opposite, the large time behaviour of solutions of the Vlasov-Poisson system is not very well understood. A scattering result due to Caglioti and Maffei [25] is more or less the unique result (in the one-dimensional periodic case) which has been obtained up to now.

The large-time asymptotics of the linearized version of the Vlasov-Poisson system is known under the name of Landau damping [32,51]. The instability of the so-called BGK waves has been studied in a series of papers by Strauss, Guo and Lin $[62,63,64,65,71]$, while the nonlinear stability has been tackled by Rein and his co-authors $[9,10,57,22]$. Also see the much more difficult case of gravitational forces $[92,94,58,60,61]$, and [23] for recent results in the presence of a confining potential. Some extensions to the case of electromagnetic forces (Vlasov-Maxwell system) are also available.

Without confinement in the whole space, dispersion effects dominate for large times and asymptotics are more or less understood $[68,86]$ although the description of the asymptotic behaviour is not very precise [48]. For bounded domains with specular reflection boundary conditions or unbounded domains with confinement $[46,78,79]$, the stability results do not provide so much information on the solutions (which are time-reversible at least for classical solutions). Injection or diffuse reflection boundary conditions introduce a source of irreversibility which is the scope of our paper. We also consider the case of compatible collision terms. By compatible, we mean that the stationary solution determined by the boundary conditions belongs to the kernel of the collision operator, if there is any. This is a severe restriction for some collision kernels like the classical Boltzmann collision operator (only maxwellian functions are allowed), a case which has been studied a long time ago, at a formal level, by Darrozès and Guiraud [31]. There are other cases where compatibility is not as much restrictive, like in the case of the elastic collision operator. In case of uncompatible boundary conditions, again very little is known. Some existence results of stationary solutions have been obtained by Arkeryd and Nouri $[89,4,3,2]$, but as far as we know, uniqueness is mainly open, and some of our results are a first step in that direction.

Technically speaking, we are going to use weak or renormalized solutions and trace properties of these solutions which have recently been studied by Mischler [41, 78, 79, 80], and entropy functionals which are very close to the ones which are used for nonlinear parabolic equations $[27,18]$. There are some deep connections between entropies for kinetic equations and for nonlinear diffusions, which are out of the scope of this paper. However, to illustrate this point, we will derive a diffusive limit, at a formal level (see [55, 74, 52, 13, 33, 91, 83] for rigorous results).

Further references corresponding to more specific aspects will be mentioned in the rest of the paper. We will not provide all details for each proof and will systematically refer to papers in which details or similar ideas can be found. Some of the results presented here have been announced in a note [12].

This paper is organized as follows. In Section 2, we will develop at a formal level a strategy to study the long time behaviour. Namely, we will prove an entropy inequality for the Vlasov-BoltzmannPoisson system with incoming boundary conditions and state its consequences on the long time behaviour and the stationary solutions. Section 3 is devoted to the application of the strategy to the various examples cited above. For uncompatible boundary conditions, the uniqueness of the stationary solutions of the equation corresponding to a special BGK approximation of the Boltzmann collision operator, when there is no self-consistent potential, and a corresponding large time convergence result 
are proved in Section 4. In Section 5, we extend the relative entropy approach to other types of boundary conditions. Technical results (proof of Theorem 2.5, statements on the Bolza problem) and general considerations (nonlinear stability, diffusive limits and relations between relative entropies for kinetic equations and for nonlinear parabolic equations) have been postponed to Appendices A-D.

\section{Strategy and results}

In this section we shall expose our strategy for the study of irreversibility and the large time asymptotics. One of the main difficulties is the lack of uniform in time estimates. For instance, the total mass is not conserved since particles are continuously injected into the domain. By introducing a relative entropy, we shall obtain a priori estimates and then use them in order to pass to the limit. All computations are done at a formal level. Rigorous proofs corresponding to the various examples of Section 1 are postponed to Section 3.

\subsection{Relative entropy and irreversibility}

Let us define the relative entropy of two functions $g, h$ of the $(x, v)$ variables by

$$
\Sigma_{\gamma}[g \mid h]=\int_{\Omega}\left(\beta_{\gamma}(g)-\beta_{\gamma}(h)-(g-h) \beta_{\gamma}^{\prime}(h)\right) d x d v+\frac{1}{2} \int_{\omega}|\nabla U[g-h]|^{2} d x,
$$

where $\beta_{\gamma}$ is the real function defined by

$$
\beta_{\gamma}(g)=-\int_{0}^{g} \gamma^{-1}(z) d z
$$

We may notice that, since $\gamma$ is strictly decreasing, the function $\beta_{\gamma}$ is strictly convex. Consequently $\Sigma_{\gamma}[g \mid h]$ is always nonnegative and vanishes if and only if $g=h$ a.e. This provides the following version of the $H$-Theorem.

Theorem 2.1 Assume that $f_{0} \in L^{1} \cap L^{\infty}$ is a nonnegative function such that $\Sigma_{\gamma}\left[f_{0} \mid M\right]<+\infty$. Let $f$ be a smooth sufficiently decaying solution of (1) and assume that $\gamma$ and $Q$ satisfy Assumptions (H1)-(H4). Then the relative entropy $\Sigma_{\gamma}[f(t) \mid M]$ where $M$ is defined by (4) satisfies

$$
\frac{d}{d t} \Sigma_{\gamma}[f(t) \mid M]=-\Sigma_{\gamma}^{+}[f(t) \mid M]-\int_{\omega} D[f](x, t) d x
$$

where $D[f]$ is defined in (2) and $\Sigma_{\gamma}^{+}$is the boundary relative entropy flux given by

$$
\Sigma_{\gamma}^{+}[g \mid h]=\int_{\Gamma^{+}}\left(\beta_{\gamma}(g)-\beta_{\gamma}(h)-(g-h) \beta_{\gamma}^{\prime}(h)\right) d \sigma .
$$

Here, smooth means for instance $C^{1}$ and sufficiently decaying means that all integrations by parts involved in the formal computation below can be done rigorously. Depending on $Q$, weaker conditions will be required for $f$ : see Section 3. For weak or renormalized solutions, the equality in (6) will be replaced by an inequality.

Proof. We first deduce from the Vlasov-Boltzmann equation (1), that for any regular function $\beta$ the following identity holds whenever all terms make sense

$$
\frac{d}{d t} \int_{\Omega} \beta(f) d x d v=\int_{\Gamma^{-}} \beta(f) d \sigma-\int_{\Gamma^{+}} \beta(f) d \sigma+\int_{\Omega} \beta^{\prime}(f) Q(f) d x d v .
$$

The second identity that we shall use is the usual energy balance: multiplying the Vlasov-Boltzmann equation by $\frac{1}{2}|v|^{2}$ and integrating with respect to $x$ and $v$ provides the identity

$$
\frac{d}{d t} \int_{\Omega} f\left(\frac{1}{2}|v|^{2}+\frac{1}{2} U[f]+\phi_{0}\right) d x d v=\sum_{ \pm} \pm \int_{\Gamma^{\mp}} f\left(\frac{1}{2}|v|^{2}+\phi_{0}\right) d \sigma+\int_{\Omega} \frac{1}{2}|v|^{2} Q(f) d x d v .
$$


We recall that the above identity requires the use of mass conservation

$$
\frac{\partial \rho}{\partial t}+\operatorname{div}_{x} j=0
$$

where $\rho(x, t)=\int_{\mathbb{R}^{d}} f(x, v, t) d v$ and $j(x, t)=\int_{\mathbb{R}^{d}} v f(x, v, t) d v$, which in turn gives

$$
\begin{aligned}
\frac{d}{d t}\left(\frac{1}{2} \int_{\Omega} f U[f] d x d v\right) & =\int_{\Omega} \frac{\partial f}{\partial t} U[f] d x d v=-\int_{\omega} \operatorname{div}_{x} j U[f] d x \\
& =\int_{\omega} j \cdot \nabla_{x} U[f] d x=-\int_{\Omega} \frac{1}{2}|v|^{2} \nabla_{x} U[f] \cdot \nabla_{v} f d x d v .
\end{aligned}
$$

Now, we notice that $\frac{1}{2}|v|^{2}+\phi_{0}(x)=-U[M]-\beta_{\gamma}^{\prime}(M): \int_{\Gamma^{ \pm}} f\left(\frac{1}{2}|v|^{2}+\phi_{0}(x)\right) d \sigma=-\int_{\Gamma^{ \pm}} f \beta_{\gamma}^{\prime}(M) d \sigma$,

$$
\text { and } \int_{\Omega} f\left(\frac{1}{2}|v|^{2}+\frac{1}{2} U[f]+\phi_{0}\right) d x d v=\int_{\Omega}\left[\frac{1}{2}(f-M) U[f-M]-\frac{1}{2} M U[M]-f \beta_{\gamma}^{\prime}(M)\right] d x d v .
$$

Taking the sum of (7) (in which $\beta=\beta_{\gamma}$ ) and (8), and noticing that

$$
\frac{d}{d t} \int_{\Omega}\left[\beta_{\gamma}(M)+M \beta_{\gamma}^{\prime}(M)+\frac{1}{2} M U[M]\right] d x d v=\sum_{ \pm} \pm \int_{\Gamma^{ \pm}}\left[\beta_{\gamma}(M)+M \beta_{\gamma}^{\prime}(M)\right] d \sigma=0
$$

we immediately (6). Of course, the contribution on $\Gamma^{-}$to the relative entropy flux vanishes since $f=M$ on this part of the boundary.

Since $\Sigma_{\gamma}[g \mid h]$ is always nonnegative, the above theorem provides a uniform in time control on $f(t)$. Like in whole space problems $[7,46,39]$, the relative entropy $\Sigma_{\gamma}[f(t) \mid M]$ provides a Lyapunov functional for the study of the large time behaviour, which can also be used to study the nonlinear stability $[92,22,23]$ (see Appendix C). An important difference with whole space problems and with previous studies of boundary value problems $[26,19]$ is that the total mass is not conserved (see Section 5 for boundary conditions preserving the mass).

\subsection{The large time limit}

Integrating the entropy dissipation inequality with respect to time provides the following inequality

$$
\Sigma_{\gamma}[f(t) \mid M]+\int_{0}^{t} \Sigma_{\gamma}^{+}[f(s) \mid M] d s+\int_{0}^{t} \int_{\omega} D\left[f(x, \cdot, s] d x d s \leq \Sigma_{\gamma}\left[f_{0} \mid M\right]\right.
$$

(this is an equality for classical solutions). Since the left hand side is the sum of three nonnegative terms (under Assumption (H4)), each of them is bounded by the right hand side. In order to investigate the large time behaviour of the solution $(f, \phi)$, we consider an arbitrary increasing and diverging sequence $\left(t_{n}\right)$ of positive real numbers and define

$$
\left(f^{n}(x, v, t), \phi^{n}(x, t)\right)=\left(f\left(x, v, t+t_{n}\right), \phi\left(x, t+t_{n}\right)\right) .
$$

It is clear from the above estimates that

$$
\lim _{n \rightarrow+\infty} \int_{\mathbb{R}^{+}} \Sigma_{\gamma}^{+}\left[f^{n}(s) \mid M\right] d s=\lim _{n \rightarrow+\infty} \int_{\mathbb{R}^{+}} \int_{\omega} D\left[f^{n}(x, \cdot, s)\right] d x d s=0
$$

and

$$
\sup _{t>0} \Sigma_{\gamma}\left[f^{n}(t) \mid M\right] \leq C .
$$

The last inequality provides a uniform in time estimate for $f^{n}$ as well as a uniform $H^{1}$ bound for $\phi^{n}$. The remainder of the method consists in proving that 
1. According to the Dunford-Pettis criterion, up to the extraction of a subsequence, $\left(f^{n}, \phi^{n}\right)$ weakly converges in $L_{\mathrm{loc}}^{1}\left(d t, L^{1}(\Omega)\right) \times L_{\mathrm{loc}}^{1}\left(d t, H_{0}^{1}(\omega)\right)$ towards a solution $\left(f^{\infty}, \phi^{\infty}\right)$ of $(1)$,

2. The limit function $f^{\infty}$ satisfies $\sup _{t \in \mathbb{R}} \Sigma_{\gamma}\left[f^{\infty}(t) \mid M\right] \leq C$ and

$$
\int_{\mathbb{R}} \Sigma_{\gamma}^{+}\left[f^{\infty}(s) \mid M\right] d s=-\int_{\mathbb{R}} \int_{\omega} D\left[f^{\infty}(x, \cdot, s)\right] d x d s=0 .
$$

Depending on the a priori estimates, (1) will be satisfied by $\left(f^{\infty}, \phi^{\infty}\right)$ either as a weak solution or even in the sense of renormalized solutions. Item 2 above allows to show that $f^{\infty}=M$ on $\Gamma^{+}$and that $Q\left(f^{\infty}\right)=0$ under Assumption (H5). Therefore $\left(f^{\infty}, \phi^{\infty}\right)$ is a solution of

$$
\left\{\begin{array}{cl}
\partial_{t} f+v \cdot \nabla_{x} f-\left(\nabla_{x} \phi+\nabla_{x} \phi_{0}\right) \cdot \nabla_{v} f=0, \quad(x, v, t) \in \Omega \times \mathbb{R}, \\
Q(f)=0, \quad & f_{\mid \Gamma \times \mathbb{R}^{+}}(x, v, t)=\gamma\left(\frac{1}{2}|v|^{2}+\phi_{0}(x)\right), \\
-\Delta \phi=\rho=\int f d v \quad & (x, t) \in \omega \times \mathbb{R}, \\
\text { and } \phi(x, t)=0, & (x, t) \in \partial \omega \times \mathbb{R}, \\
\sup _{t \in \mathbb{R}} \Sigma_{\gamma}[f(t) \mid M] \leq C . &
\end{array}\right.
$$

Notice that the time variable $t$ lies in the whole real line and that the boundary conditions on $f^{\infty}$ are overdetermined, since $f^{\infty}$ is given on the whole boundary $\Gamma$ and not only on $\Gamma^{-}$. A second source of overdetermination for the system (11) is the condition $Q\left(f^{\infty}\right)=0$ (when $Q$ is not identically vanishing).

As we shall see in Section 3, this program can be completed for each of the examples of Section 1. When $Q \equiv 0, Q=Q_{E}$ or $\alpha \neq 0$ in Examples 2, 3 and 5, if $f_{0}$ is bounded in $L^{\infty}, f(t)$ is also uniformly bounded in $L^{\infty}$, and we may easily pass to the limit. The other examples (including the case $\alpha=0$ ) require additional work (using for instance renormalized solutions). Up to this question which is a little bit delicate, the irreversibility result of Theorem 2.1 provides a characterization of the large time limit that we can summarize in the following formal result (it is formal in the sense that we assume the convergence of the collision term, which is a property that has to be proved case by case).

Corollary 2.2 Assume that $f_{0} \in L^{1} \cap L^{\infty}$ is a nonnegative function such that $\Sigma_{\gamma}\left[f_{0} \mid M\right]<+\infty$. Under Assumptions (H1)-(H5), consider an unbounded increasing sequence $\left(t_{n}\right)_{n \in \mathbb{N}}$. If $\left(f^{n}, \phi^{n}\right)$ defined by (10) weakly converges to some $\left(f^{\infty}, \phi^{\infty}\right)$ in $L_{\mathrm{loc}}^{\infty}\left(d t, L^{1}(\Omega)\right) \times L_{\mathrm{loc}}^{\infty}\left(d t, H_{0}^{1}(\omega)\right)$ and if $Q\left(f^{n}\right) \stackrel{\mathcal{D}^{\prime}}{\rightarrow} Q\left(f^{\infty}\right)$, then $\left(f^{\infty}, \phi^{\infty}\right)$ is a solution of (11) (which belongs to the kernel of $Q$ for any $(t, x) \in \mathbb{R} \times \omega$ and is such that $f_{\mid \Gamma^{+}}(x, v, t)=\gamma\left(|v|^{2} / 2+\phi_{0}(x)\right)$ for any $\left.t \in \mathbb{R}^{+},(x, v) \in \Gamma^{+}\right)$.

Proof. We have to prove the convergence of $\nabla_{x} \phi^{n} \cdot \nabla_{v} f^{n}$ to $\nabla_{x} \phi^{n} \cdot \nabla_{v} f^{n}$ as $n \rightarrow+\infty$. If $f^{n}$ is uniformly bounded in $L^{\infty}$, by interpolation (see [73, 67]) with the kinetic energy, $\rho^{n}=\int_{\mathbb{R}^{d}} f^{n} d v$ is bounded in $L^{\infty}\left(d t, L^{q}\left(\mathbb{R}^{d}\right)\right)$ with $q=1+2 / d$. Using the compactness properties of $\nabla \Delta^{-1}$, it is easy to pass to the limit in the self-consistent term.

Without uniform bounds, one uses renormalized solutions [41, 78, 79, 80] and (11) only holds in the renormalized sense (compactness for $\rho^{n}$ is a consequence of averaging lemmas).

\subsection{Are the solutions of the limit problem stationary?}

In this paragraph, we provide some rigorous results ensuring the stationarity of the solutions of the limit problem (11). If $f^{\infty} \in \operatorname{Ker} \mathrm{Q}$ depends only on $|v|^{2}$ (examples 2, 3 and 4), we apply the following

Lemma 2.3 Let $f \in L_{l o c}^{1}$ be a solution of the Vlasov equation in the renormalized sense. If $f$ is even (or odd) with respect to the $v$ variable, then it does not depend on $t$. 
The proof is straightforward. The operator $\partial_{t}$ conserves the $v$ parity while $v \cdot \nabla_{x}-\left(\nabla_{x} \phi+\nabla_{x} \phi_{0}\right) \cdot \nabla_{v}$ transforms the $v$ parity into its opposite.

Corollary 2.4 Let $f$ be a solution of (11) with $Q=Q_{E}, Q_{F P, \alpha}, Q_{\alpha}, Q_{e e, \alpha}+Q_{E}, Q_{e e, \alpha}+Q_{\alpha}$, $Q_{e e, \alpha}+Q_{F P, \alpha}$ or a linear combination of these operators (with nonnegative coefficients). Then $f$ does not depend on $t$, and is nothing else than the function $M$ defined in (4) under the additional assumption that there are no closed characteristics if $Q=Q_{E}$.

The proof is an immediate application of Lemma 2.3. Indeed, using the $H$-Theorem, we deduce that the kernel of a (nonnegative) linear combination of the above collision operators is equal to the intersection of the kernels. Therefore, any function $f$ satisfying $Q(f)=0$ is even with respect to $v$. The assumption that there are no closed characteristics means that any characteristics is connected to the boundary, which proves that $f \equiv M$.

If $d=1$, a sufficient condition to avoid closed characteristics is the condition $-\frac{d^{2} \phi_{0}}{d x^{2}} \geq 0$. For the pure Vlasov-Poisson system $(Q \equiv 0)$ proving that $f^{\infty}$ is stationary is an interesting open problem. It is true (when $d=1$ ) if the potential is analytic:

Theorem 2.5 Assume that $\gamma$ satisfies Property $(\mathcal{P})$ and consider a solution $(f, \phi)$ of the limit problem (11) (with $Q \equiv 0$ ) on the interval $\omega=(0,1)$. If $\phi_{0}$ is analytic in $x$ with $C^{\infty}$ (in time) coefficients and if $\phi_{0}$ is analytic with $-\frac{d^{2} \phi_{0}}{d x^{2}} \geq 0$ on $\omega$, then $(f, \phi)$ is the unique stationary solution, given by: $f=M, \phi=U[M]$.

Proof. Without loss of generality, we assume that $\phi_{0}(0)=0$ and $\phi_{0}(1) \geq 0$ in such a way that $\phi_{0}^{\prime}(0) \geq 0$. Defining the characteristics $t \mapsto(X, V)(t)=(X, V)(t ; x, v, s)$ as the unique solutions of

$$
\begin{array}{ll}
\frac{\partial X}{\partial t}=V, & \frac{\partial V}{\partial t}=-\frac{\partial \phi}{\partial x}(X, t)-\frac{d \phi_{0}}{d x}(X), \\
X(s ; x, v, s)=x, & V(s ; x, v, s)=v,
\end{array}
$$

corresponding to a given $(s ; x, v) \in \mathbb{R} \times \Omega$, there exists an interval $\left(\mathcal{T}_{\text {in }}(s ; x, v), \mathcal{T}_{e}(s ; x, v)\right)$ on which the characteristics are defined. This interval is such that:

- $\mathcal{T}_{\text {in }}(s ; x, v)=-\infty$ or $\left(X_{i n}, V_{i n}\right)(s ; x, v):=(X, V)\left(\mathcal{T}_{\text {in }}(s ; x, v) ; x, v, s\right) \in \Gamma^{-}$,

- $\mathcal{T}_{e}(s ; x, v)=+\infty$ or $\left(X_{e}, V_{e}\right)(s ; x, v):=(X, V)\left(\mathcal{T}_{e}(s ; x, v) ; x, v, s\right) \in \Gamma^{+}$.

Step 1 : the electric field is repulsive at $x=0$. Along the characteristics, the total energy satisfies

$$
\frac{\partial}{\partial t}\left[\frac{1}{2}|V|^{2}+\phi(X, t)+\phi_{0}(X)\right]=\frac{\partial \phi}{\partial t}(X, t) .
$$

As a consequence, there exists $v_{M}>0$ depending on $\left\|\phi+\phi_{0}\right\|_{L^{\infty}}$ and $\left\|\partial_{t} \phi\right\|_{L^{\infty}}$ such that the following properties hold for $v>v_{M}$

- for all $x \in(0,1)$ and $s \in \mathbb{R},-\infty<\mathcal{T}_{\text {in }}(s ; x, v)<s<\mathcal{T}_{e}(s ; x, v)<+\infty$,

- $X_{\text {in }}(s ; x, v)=0$,

- $\left|V_{i n}(s ; x, v)\right|^{2} \leq|v|^{2}+2 C_{M}$ where $C_{M}$ only depends on $v_{M}$.

We claim that this ensures the existence of a constant $C_{1}>0$ such that $\rho(x, t)=\int_{\mathbb{R}^{d}} f(x, v, t) d v \geq C_{1}$, which implies, thanks to the Poisson equation, the existence of a positive constant $C_{2}>0$ such that for any $t \in \mathbb{R}$,

$$
\frac{\partial \phi}{\partial x}(0, t) \geq C_{2}
$$


To prove our claim, we first deduce from the Vlasov equation and the boundary condition that

$$
f(x, v, t)=f\left(0, V_{i n}(x, v, t), \mathcal{T}_{i n}(x, v, t)\right)=\gamma\left(\frac{1}{2}\left|V_{i n}(x, v, t)\right|^{2}\right) .
$$

In view of the above estimates on $V_{i n}$ and due to the decay of $\gamma$, we get the estimate

$$
\rho(x, t) \geq \int_{v_{M}}^{+\infty} \gamma\left(\frac{1}{2}|v|^{2}+C_{M}\right) d v=: C_{1}>0
$$

The conclusion then holds with $C_{2}=\frac{1}{2} C_{1}$ using

$$
0=-\int_{0}^{1} \frac{\partial \phi}{\partial x}(x, t) d x \geq\left(-\frac{\partial \phi}{\partial x}(0, t) x+C_{1} \frac{x^{2}}{2}\right)_{\mid x=1} .
$$

Step 2 : Analysis of the characteristics in a neighborhood of $(0,0, t)$. Since the electric field $\frac{\partial \phi}{\partial x}$ is (uniformly in $t)$ positive in a neighborhood of $x=0_{+}$, there exists $x_{M} \in(0,1)$ such that for every $x_{0} \in\left(0, x_{M}\right)$ and every $t_{0} \in \mathbb{R}$

$$
-\infty<\mathcal{T}_{\text {in }}\left(t_{0}, x_{0}, 0\right)<t_{0}<\mathcal{T}_{e}\left(t_{0}, x_{0}, 0\right)<+\infty \quad \text { and } \quad X_{\text {in }}\left(t_{0}, x_{0}, 0\right)=X_{e}\left(t_{0}, x_{0}, 0\right)=0 .
$$

Saying that $f$ is constant along the characteristics means $f\left(X_{i n}, V_{i n}, \mathcal{T}_{\text {in }}\right)=f\left(X_{e}, V_{e}, \mathcal{T}_{e}\right)$. Besides, we deduce from the boundary conditions (11) that

$$
f\left(X_{i n}, V_{i n}, \mathcal{T}_{i n}\right)=\gamma\left(\frac{1}{2}\left|V_{i n}\right|^{2}\right), \quad f\left(X_{e}, V_{e}, \mathcal{T}_{e}\right)=\gamma\left(\frac{1}{2}\left|V_{e}\right|^{2}\right) .
$$

Since $\gamma$ is strictly decreasing, this yields

$$
\left|V_{\text {in }}\left(t_{0}, x_{0}, 0\right)\right|=\left|V_{e}\left(t_{0}, x_{0}, 0\right)\right| \quad \forall\left(x_{0}, t_{0}\right) \in\left(0, x_{M}\right) \times \mathbb{R} .
$$

We claim that this is enough to ensure that $\phi$ is stationary (see Corollary A.2 in Appendix A). The existence and uniqueness results for the solutions of the stationary Vlasov-Poisson system when $-\partial_{x x} \phi_{0} \geq 0$ can be found in [56].

Analyticity results are available only for whole space or periodic evolution problems [14] (note that analyticity is the standard framework for the study of the Landau damping [25, 32, 51]). We are not aware of any analyticity result for boundary value problems. In the above theorem, the assumption on $\phi_{0}$ is made only to avoid closed characteristics [89].

\subsection{A uniqueness result for the BGK approximation of the Boltzmann operator}

In this paragraph, we shall only consider the case of the BGK approximation $Q_{\alpha}(\alpha \geq 0)$ of the Boltzmann collision operator for fermions, in the case of a boundary condition $g$ which is not necessarily compatible, in the sense of Assumptions (H4)-(H5), with $Q=Q_{\alpha}$ and without self-consistent potential:

$$
\begin{aligned}
& v \cdot \nabla_{x} f-\nabla_{x} \phi_{0} \cdot \nabla_{v} f=Q_{\alpha}(f) \quad \forall(x, v) \in \Omega \\
& f(x, v)=g(x, v) \quad \forall(x, v) \in \Gamma^{-}
\end{aligned}
$$

has a solution such that $0 \leq f(x, v) \leq F_{D}(x, v)=\left(\alpha+e^{\left(\frac{1}{2}|v|^{2}+\phi_{0}(x)-\mu\right) / \theta}\right)^{-1}$ as soon as

$$
0 \leq g \leq F_{D} \quad \text { on } \quad \Gamma^{-}
$$

(see [87] in the case $\alpha=0$, [84] if $\alpha>0$ ). With relative entropy methods, one may first prove a uniqueness result whose proof is inspired by the arguments developed in $[87,84]$. 
Theorem 2.6 Assume that $\alpha \geq 0$ and consider two nonnegative solutions $f_{1}$ and $f_{2}$ of (13) such that for any $(x, v) \in \Omega, f_{i}(x, v) \leq F_{D}(x, v)=\left(\alpha+e^{\left(\frac{1}{2}|v|^{2}+\phi_{0}(x)-\mu\right) / \theta}\right)^{-1}$ (for $\left.i=1,2\right)$. Then $f_{1}=f_{2}$.

Note here that we do not make any assumption on $\phi_{0}$ saying for instance that there are no closed characteristics. The proof of Theorem 2.6 is deferred to Section 4 . Let us denote by $f_{s}$ the unique stationary solution of (13). A computation similar to the one of Theorem 2.6 provides the following result on large time asymptotics.

Theorem 2.7 Assume that $\alpha \geq 0$. If (14) is satisfied, then any solution of

$$
\begin{aligned}
& \partial_{t} f+v \cdot \nabla_{x} f-\nabla_{x} \phi_{0} \cdot \nabla_{v} f=Q_{\alpha}(f), \quad t>0, \quad(x, v) \in \Omega \\
& f(x, v)=g(x, v), \quad(x, v) \in \Gamma^{-}
\end{aligned}
$$

with an intial data $f_{0}$ such that $0 \leq f_{0} \leq F_{D} *$-weakly converges in $L^{\infty}(\Omega)$, as time tends to $+\infty$, towards the unique stationary solution $f_{s}$ of (13).

\section{Application to the examples of Section 1}

This section is devoted to proofs of the results on large time limits (without the formal assumptions of Corollary 2.2) and on the limiting solution (Corollary 2.4) in each of the Examples of Section 1. Details are given for the pure Vlasov-Poisson system (Example 1), the case of a linear elastic collision operator (Example 4) and the BGK approximation of the Boltzmann operator (Example 3). Only brief indications and references to the existing literature are given for the Vlasov-Poisson-Fokker-Planck system (Example 2) and the case of an Electron-Electron collision operator (Example 5), which are (at least for $\alpha=0$ ) more standard.

\subsection{Examples 1 and 4: no collision or elastic collisions}

We treat in the same way Examples 1 and 4 because the choice of $\gamma$ is arbitrary in both cases. Note that the solution $f$ of the time dependent problem satisfies a maximum principle in both cases: if $f_{0}$ is bounded, then $f(t)$ is bounded as well according to $\sup |f(t)| \leq \max \left(\sup \left|f_{0}\right|, \sup \gamma\right)$. The $L^{\infty}$ bound will be useful for passing to the limit. Throughout this section, we shall assume that

$$
f_{0} \in L_{x, v}^{1} \cap L_{x, v}^{\infty}, \quad|v|^{2} f_{0} \in L_{x, v}^{1} \quad \text { and } \quad \Sigma_{\gamma}\left[f_{0} \mid M\right]<+\infty .
$$

Moreover, we require that

(H7)

$$
\int_{0}^{+\infty} s^{(d+1) / 2} \gamma(s) d s<+\infty,
$$

so that $\int_{\mathbb{R}^{d}}|v|^{3} \gamma\left(|v|^{2}\right) d v$ makes sense, and we assume that

(H8)

$$
\gamma^{\prime} \text { is bounded on }[-A,+\infty) \text { for any } A>0 \text {. }
$$

We shall first prove two preliminary results and then state a theorem which covers the results of Theorem 2.1 and Corollary 2.2 at once.

Lemma 3.1 Under Assumptions (H6)-(H8), there exists a positive constant $C_{A}$ such that for any $(u, v) \in[0, A]^{2}$,

$$
\begin{aligned}
& \beta_{\gamma}(u)-\beta_{\gamma}(v)-\beta_{\gamma}^{\prime}(v)(u-v) \geq \frac{1}{2} C_{A}|u-v|^{2}, \\
& \text { and } \quad\left(\gamma^{-1}(u)-\gamma^{-1}(v)\right)(v-u) \geq C_{A}|u-v|^{2} .
\end{aligned}
$$


Proof. Since $-\left(\gamma^{-1}\right)^{\prime}(u)=-\left(\gamma^{\prime} \circ \gamma^{-1}(u)\right)^{-1}$ and $-\gamma^{\prime}(u) \leq C$ according to (15), we have, for any $u \in[0, A],-\left(\gamma^{-1}\right)^{\prime}(u) \geq 1 / C$. Consequently,

$$
\begin{aligned}
& \beta_{\gamma}(u)-\beta_{\gamma}(v)-\beta_{\gamma}^{\prime}(v)(u-v)=-\int_{v}^{u}\left(\int_{v}^{t}\left(\gamma^{-1}\right)^{\prime}(s) d s\right) d t \geq \frac{1}{2 C}|v-u|^{2} . \\
& \text { and } \quad\left(\gamma^{-1}(u)-\gamma^{-1}(v)\right)(u-v)=\frac{\gamma^{-1}(u)-\gamma^{-1}(v)}{v-u}(v-u)^{2} \geq \frac{1}{C}|v-u|^{2} .
\end{aligned}
$$

which proves the result with $C_{A}=1 / C$.

Corollary 3.2 Let $A>0$. Under Assumptions (H6)-(H8), there exists a positive constant $C_{A}$ such that for all $f, g \in L_{x, v}^{2}(\Omega)$ with $0 \leq f \leq A$ and $0 \leq g \leq A$ a.e., we have:

(i) $\Sigma_{\gamma}[f \mid g] \geq C_{A}\|f-g\|_{L_{x, v}^{2}(\Omega)}^{2}$

(ii) $\Sigma_{\gamma}^{+}[f \mid g] \geq C_{A}\|f-g\|_{L^{2}\left(\Gamma^{+}, d \sigma\right)}^{2}$

(iii) Assume that $\chi$ is symmetric, measurable, positive a.e. on $\left\{\left(v, v^{\prime}\right) \in \mathbb{R}^{d} \times \mathbb{R}^{d}:|v|=\left|v^{\prime}\right|\right\}$, and consider the linear elastic collision operator $Q_{E}$ defined by (5). Then

$$
D[f] \geq \frac{1}{2}\left|S^{d-1}\right| C_{A} \iint_{\mathbb{R}^{d} \times S^{d-1}}|v|^{d-2} \chi(v,|v| \omega)|f(v)-f(|v| \omega)|^{2} d \omega d v,
$$

where $d \omega$ is the measure induced by Lebesgue's measure on the unit sphere $S^{d-1}$ of $\mathbb{R}^{d}$.

Proof. (i) and (ii) are straightforward consequences of Lemma 3.1. To prove (iii), we use again Lemma 3.1, applied to

$$
D[f]=-\int Q_{E}(f) \gamma^{-1}(f) d v=-\frac{1}{2} \int_{\mathbb{R}^{d} \times \mathbb{R}^{d}} \chi\left(v, v^{\prime}\right) \delta\left(|v|^{2}-\left|v^{\prime}\right|^{2}\right)\left(f-f^{\prime}\right)\left(\gamma^{-1}(f)-\gamma^{-1}\left(f^{\prime}\right)\right) d v d v^{\prime} .
$$

These estimates allow us to prove rigourously a result on the large time behaviour for $Q_{E}$. The existence of solutions can be found in $[11,1,78,79,80]$. In these references, stability results are also proved for renormalized solutions.

Theorem 3.3 Assume that (H6)-(H8) hold and that $\chi$ is symmetric, measurable, nonnegative. The Vlasov-Poisson-Boltzmann system (1) with $Q=Q_{E}$ or $Q=0$ admits a weak solution $f \in L^{\infty}\left(\mathbb{R}^{+} \times \Omega\right)$ such that

$$
\|f(\cdot, \cdot, t)\|_{L^{\infty}} \leq \max \left(\left\|f_{0}\right\|_{L^{\infty}, \gamma}\left(\inf _{\partial \omega} \phi_{0}\right)\right) .
$$

The sequence $\left(f^{n}, \phi^{n}\right)$ defined by (10) converges up to the extraction of a subsequence, *-weakly in $L^{\infty}\left(\mathbb{R}^{+} \times \Omega\right) \times L_{\mathrm{loc}}^{\infty}\left(\mathbb{R}^{+}, H_{0}^{1}(\Omega)\right)$, towards a solution $\left(f_{\infty}, \phi_{\infty}\right)$ of $(11)$. In case $Q=Q_{E}$, if $v \mapsto$ $\inf _{v \in \mathbb{R}^{d}} \int_{|\omega|=1}|v|^{d-2} \chi(v,|v| \omega) d \omega$ is positive a.e., then $\left(f_{\infty}, \phi_{\infty}\right)$ is stationary.

Proof. The $L^{\infty}$ estimate is straightforward in case $Q=0$. For $Q=Q_{E}$, we may use the fact that $Q_{E}(1)=0$ and use the decomposition $Q_{E}(f)=Q_{E}^{+}(f)-\lambda f$ where

$$
Q^{+}(f)=|v|^{d-2} \int_{S^{d-1}} \chi(v,|v| \omega) f(|v| \omega) d \omega \quad \text { and } \quad \lambda=|v|^{d-2} \int_{S^{d-1}} \chi(v,|v| \omega) d \omega=Q^{+}(1) .
$$

The existence proof of $f$ goes as follows. For a given $\phi$, we remark that $f$ has to solve

$$
\partial_{t} f+v \cdot \nabla_{x} f-\left(\nabla_{x} \phi+\nabla_{x} \phi_{0}\right) \cdot \nabla_{v} f+\lambda f=Q^{+}(f) .
$$


The mapping $f \mapsto g$ defined by

$$
\left\{\begin{array}{l}
\partial_{t} g+v \cdot \nabla_{x} g-\left(\nabla_{x} \phi+\nabla_{x} \phi_{0}\right) \cdot \nabla_{v} g+\lambda g=Q^{+}(f), \\
g_{\mid t=0}=f_{0}, \quad g_{\mid \Sigma^{-}}=\gamma\left(\frac{1}{2}|v|^{2}+\phi_{0}\right) .
\end{array}\right.
$$

is contractive in $L^{\infty}((0, T) \times \Omega)$. It has a unique fixed point which can be computed with an iteration scheme. Starting the iteration procedure from a nonnegative initial point, the Maximum Principle is satisfied at each step, which implies that the solution $f$ is nonnegative if $f_{0} \geq 0$ and $\gamma \geq 0$. On the other hand, for any $K \in \mathbb{R}, K-f$ also satisfies the same equation with $f_{0}$ and $\gamma\left(\frac{1}{2}|v|^{2}+\phi_{0}\right)$ replaced by $K-f_{0}$ and $K-\gamma\left(\frac{1}{2}|v|^{2}+\phi_{0}\right)$ respectively, which proves the Maximum Principle for a solution of $(1)$.

Let $\left(f_{n}(x, v, t), \phi_{n}(x, t)\right)=\left(f\left(x, v, t+t_{n}\right), \phi\left(x, t+t_{n}\right)\right)$ with $\lim _{n \rightarrow+\infty} t_{n}=+\infty$ and consider the limit as $n \rightarrow+\infty$. According to (9),

$$
\begin{aligned}
& \lim _{n \rightarrow+\infty} \int_{0}^{+\infty} \Sigma_{\gamma}^{+}\left[f_{n}(\cdot, \cdot, t) \mid M\right] d t=0, \quad \lim _{n \rightarrow+\infty} \int_{0}^{+\infty} \int_{\omega} D\left[f_{n}(x, \cdot, t)\right] d x d t=0 \quad \text { if } \quad Q=Q_{E}, \\
& \sup _{t \in \mathbb{R}^{+}} \Sigma_{\gamma}\left[f_{n}(\cdot, \cdot, t) \mid M\right] \leq C \quad \text { and } \quad\left\|f_{n}\right\|_{L^{\infty}} \leq C .
\end{aligned}
$$

Using the stability result in [78], up to the extraction of a subsequence,

$$
\begin{array}{ll}
f_{n} \rightarrow f_{\infty} & \text { weak-* in } L^{\infty}\left(\mathbb{R}^{+} \times \Omega\right) \\
\phi_{n} \rightarrow \phi_{\infty} & \text { weak-* in } L_{\text {loc }}^{\infty}\left(\mathbb{R}^{+}, H_{0}^{1}\left(\mathbb{R}_{x}^{d}\right)\right)
\end{array}
$$

and $\left(f_{\infty}, \phi_{\infty}\right)$ is a solution of the Vlasov-Poisson-Boltzmann system. It follows from Corollary 3.2, (ii) and (iii), that

$$
\begin{aligned}
& \lim _{n \rightarrow+\infty} \int_{0}^{+\infty} \int_{\omega} \int_{S^{d-1}}|v|^{d-2} \chi(v,|v| \omega)\left|f_{n}(x, v, t)-f_{n}(x,|v| \omega, t)\right|^{2} d \omega d x d v d t=0 \\
& \text { and } \lim _{n \rightarrow+\infty} \int_{0}^{+\infty}\left\|f_{n}-M\right\|_{L_{\Gamma}^{2}}^{2} d t=0,
\end{aligned}
$$

which implies that $f_{\infty \mid \Gamma^{+}}=M_{\mid \Gamma^{+}}$and $f_{\infty}(v)=f_{\infty}(|v| \omega)$ for $\omega \in S^{d-1}$. We conclude the proof of Theorem 3.3 by applying Lemma 2.3 .

\subsection{Example 2: the linear Fokker-Planck operator}

We consider the linear Fokker-Planck operator (corresponding to $\alpha=0$ ):

$$
Q_{F P, 0}(f)=\operatorname{div}_{v}\left(v f+\theta \nabla_{v} f\right) .
$$

In the whole space case, the corresponding evolution equation has been analyzed in [20] and the large time asymptotics have been studied in details in $[21,46]$. The existence results have been adapted to bounded domains in [80], where further stability results are proved. These stability results allow us to construct a renormalized solution of the Vlasov-Poisson-Fokker-Planck equation which satisfies the integrated in time entropy inequality. Since the function $\gamma$ is given by $\gamma(u)=C e^{-u / \theta}$ and since the following inequality holds

$$
\int Q_{F P}(f) \log \left(\frac{f}{M_{\theta}}\right) d v=-\int \frac{1}{\theta f}\left|v f+\theta \nabla_{v} f\right|^{2} d v
$$

the entropy inequality takes the form

$$
\Sigma_{\gamma}[f(t) \mid M]+\int_{0}^{t} \Sigma_{\gamma}^{+}[f(s) \mid M] d s+\int_{0}^{t} \int_{\Omega} \frac{1}{\theta f}\left|v f+\theta \nabla_{v} f\right|^{2} d v d x d s \leq \Sigma_{\gamma}\left[f_{0} \mid M\right]
$$


where $\Sigma_{\gamma}[f \mid g]=\theta \int_{\Omega}\left[f \log \left(\frac{f}{g}\right)-f+g\right] d x d v+\frac{1}{2}|\nabla U[f-g]|^{2} d x$. In [80], it is proved that any sequence of renormalized solutions of the Vlasov-Poisson-Fokker-Planck system which satisfies the above entropy inequality has a subsequence which weakly converges in $L^{1}$ towards a renormalized solution of the same system. We apply this stability result to a sequence $f_{n}(x, v, t)=f\left(x, v, t+t_{n}\right)$. Then, we have a uniform bound in $L^{1}$ for $f_{n}$. Besides, a Cauchy Schwartz inequality leads to

$$
\int_{0}^{+\infty} \frac{\left(\int_{\Omega}\left|v f_{n}+\theta \nabla_{v} f_{n}\right| d v d x\right)^{2}}{\int_{\Omega} \theta f_{n} d v d x} d s \leq \int_{0}^{+\infty} \int_{\Omega} \frac{1}{\theta f_{n}}\left|v f_{n}+\theta \nabla_{v} f_{n}\right|^{2} d v d x d s=\int_{t_{n}}^{+\infty} \int_{\Omega} \frac{1}{\theta f}\left|v f+\theta \nabla_{v} f\right|^{2} d v d x d s
$$

which proves that $\lim _{n \rightarrow+\infty}\left\|v f_{n}(\cdot, s)+\theta \nabla_{v}(\cdot, s) f_{n}\right\|_{L^{1}(\Omega)}^{2}=0$. This shows that the weak limit $f_{\infty}$ of a converging subsequence is a Maxwellian: $f_{\infty}=\rho(x, t) M_{\theta}(v)$. Exactly as in [21], $f_{\infty}$ is a renormalized solution of the Vlasov-Poisson-Fokker-Planck system whose unique Maxwellian solution is given by (4) with $\gamma(u)=C e^{-u / \theta}$. Thus we obtain the

Theorem 3.4 Consider the problem (1) with $Q=Q_{F P}$. Under Assumptions (H1), (H2) and (H6), the sequence $\left(f_{n}\right)$ weakly converges in $L^{\infty}\left((0, T), L_{x, v}^{1}\right)$ towards the unique sationary solution $M$ defined by (4).

\subsection{Example 3: semiconductor BGK model}

In this paragraph, we are going to give detailed estimates which allow us to prove directly that the large time limit is in the kernel of the collision operator, without proving the convergence of $Q_{\alpha}\left(f^{n}\right)$ to $Q_{\alpha}\left(f^{\infty}\right)$ in $\mathcal{D}^{\prime}$. We deal either with the standard BGK model $(\alpha=0)$ or with the BGK model for fermions $(\alpha>0)$ :

$$
Q_{\alpha}(f)=\int_{\mathbb{R}^{d}} \sigma\left(v, v^{\prime}\right)\left[M_{\theta}(v) f\left(v^{\prime}\right)(1-\alpha f(v))-M_{\theta}\left(v^{\prime}\right) f(v)\left(1-\alpha f\left(v^{\prime}\right)\right)\right] d v^{\prime},
$$

where $\sigma$ is such that there exists two positive constants $\sigma_{0}$ and $\sigma_{1}$ for which

$$
\sigma_{0} \leq \sigma \leq \sigma_{1} .
$$

Existence results are known and we may recall the following result (the cases $\alpha=0$ and $\alpha>0$ have been treated in [77] and [81, 82] respectively).

Theorem 3.5 Let $\alpha \in \mathbb{R}^{+}$and $f_{0}$ be a $L^{1}(\Omega)$ nonnegative function such that $0 \leq f_{0} \leq \alpha^{-1}$ a.e. and $\Sigma_{\gamma}\left[f_{0} \mid M\right]<+\infty$. Then the Vlasov-Poisson-Boltzmann equation (1) with $Q=Q_{\alpha}$ admits a renormalized solution $f$ such that $0 \leq f \leq \alpha^{-1}$ a.e.

Also recall that $\gamma(u)=\left(\alpha+e^{(u-\mu) / \theta}\right)^{-1}$ and $\gamma^{-1}(s)=\mu-\theta \log \left(\frac{s}{1-\alpha s}\right)$. With these notations, the integrand of the relative entropy takes the form

$$
\beta_{\gamma}(u)-\beta_{\gamma}(v)-\beta_{\gamma}^{\prime}(v)(u-v)=\theta \int_{v}^{u}\left(\int_{v}^{s} \frac{d t}{t(1-\alpha t)}\right) d s .
$$

Lemma 3.6 Let $(u, v) \in\left(0, \alpha^{-1}\right)^{2}$. Then

$$
\beta_{\gamma}(u)-\beta_{\gamma}(v)-\beta_{\gamma}^{\prime}(v)(u-v) \geq \theta\left(u \log \left(\frac{u}{v}\right)-u+v\right) .
$$

This lemma is a straightforward consequence of the identity $\frac{1}{t(1-\alpha t)} \geq \frac{1}{t}$ which is verified for any $t \in\left(0, \alpha^{-1}\right)$. Note that if $f$ is a solution of the Vlasov-Poisson-Boltzmann equation (1) with $Q=Q_{\alpha}$ and if $f_{n}$ is defined as above, for any $t>0$, we may use the Csiszár-Kullback inequality to obtain:

$$
\Sigma_{\gamma}\left[f_{n}(t) \mid M\right] \geq \theta\left\|f_{n}(t)\right\|_{L^{1}(\Omega)} \log \left(\frac{\left\|f_{n}(t)\right\|_{L^{1}(\Omega)}}{\|M\|_{L^{1}(\Omega)}}\right)+\frac{\theta}{4\left\|f_{n}(t)\right\|_{L^{1}(\Omega)}}\left\|f-\frac{\left\|f_{n}(t)\right\|_{L^{1}(\Omega)}}{\|M\|_{L^{1}(\Omega)}} \cdot M\right\|_{L^{1}(\Omega)}^{2}
$$


where $f_{n}(t)$ stands for $f_{n}(\cdot, \cdot, t)$. A similar formula holds for $\Sigma_{\gamma}^{+}$on the boundary. The relative entropy and the relative entropy flux control the distance between $f$ and $M$ in $L^{1}(\Omega)$ and in $L^{1}\left(\Gamma^{-}\right)$ respectively.

Lemma 3.7 There exists a positive constant $C>0$ such that for any nonnegative measurable function $f$ on $\Omega$ such that $f \leq \alpha^{-1}$ a.e., the following estimate holds:

$$
\int_{\omega} D[f](x) d x \geq \frac{C}{\|f\|_{L^{1}(\Omega)}}\left(\int_{\Omega}\left|f\left(1-\alpha f^{\prime}\right) M_{\theta}^{\prime}-f^{\prime}(1-\alpha f) M_{\theta}\right| d x d v\right)^{2} .
$$

Proof. Using the fact that $\sigma\left(v, v^{\prime}\right) \geq 4 C>0$, let us compute $\frac{1}{\theta} \int_{\omega} D[f](x) d x$.

$$
-\int_{\Omega} Q_{\alpha}(f) \log g d x d v \geq 4 C \int_{\Omega \times \mathbb{R}^{d}} M_{\theta} M_{\theta}^{\prime}(1-\alpha f)\left(1-\alpha f^{\prime}\right)\left(g-g^{\prime}\right)\left(\log g-\log g^{\prime}\right) d x d v d v^{\prime}
$$

where $g=\frac{f}{(1-\alpha f) M_{\theta}}$. Using the identity

$$
(a-b)(\log a-\log b) \geq(\sqrt{a}-\sqrt{b})^{2} \geq \frac{(a-b)^{2}}{2(a+b)}
$$

for any $a, b>0$, we get

$$
\begin{aligned}
\int_{\omega} D[f(x, \cdot)] d x & \geq 2 C \int_{\Omega \times \mathbb{R}^{d}} \frac{M_{\theta} M_{\theta}^{\prime}(1-\alpha f)\left(1-\alpha f^{\prime}\right)\left(g-g^{\prime}\right)^{2}}{g+g^{\prime}} d x d v d v^{\prime} \\
& \geq 2 C \int_{\Omega \times \mathbb{R}^{d}} \frac{\left(f\left(1-\alpha f^{\prime}\right) M_{\theta}^{\prime}-f^{\prime}(1-\alpha f) M_{\theta}\right)^{2}}{f\left(1-\alpha f^{\prime}\right) M_{\theta}^{\prime}+f^{\prime}(1-\alpha f) M_{\theta}} d x d v d v^{\prime} .
\end{aligned}
$$

Besides, the Cauchy-Schwarz inequality: $\int\left(X^{2} / Y\right) \geq\left(\int|X|\right)^{2} /\left(\int Y\right)$ leads to

$$
\int_{\omega} D[f(x, \cdot)] d x \geq 2 C \frac{\left(\int_{\Omega \times \mathbb{R}^{d}}\left|f\left(1-\alpha f^{\prime}\right) M_{\theta}^{\prime}-f^{\prime}(1-\alpha f) M_{\theta}\right| d x d v d v^{\prime}\right)^{2}}{\int_{\Omega \times \mathbb{R}^{d}}\left(f\left(1-\alpha f^{\prime}\right) M_{\theta}^{\prime}+f^{\prime}(1-\alpha f) M_{\theta}\right) d x d v d v^{\prime}}
$$

Using the estimates $0 \leq 1-\alpha f \leq 1$ and $\int_{\mathbb{R}^{d}} M_{\theta} d v=1$, we find

$$
\int f\left(1-\alpha f^{\prime}\right) M_{\theta}^{\prime} d x d v d v^{\prime} \leq\|f\|_{L^{1}},
$$

which proves that the above denominator is bounded by $2\|f\|_{L^{1}}$ and the Lemma is proved.

We can now rephrase Corollary 2.2 as follows.

Theorem 3.8 Under Assumptions (H1)-(H2), consider an unbounded increasing sequence $\left(t_{n}\right)_{n \in \mathbb{N}}$ and the solution $f$ of Theorem 3.5. Then $\left(f^{n}, \phi^{n}\right)$ defined by (10) weakly converges in $L^{1}\left(\mathbb{R}_{\mathrm{loc}}^{+} \times \Omega\right)$ and $*$-weakly converges in $L_{\mathrm{loc}}^{\infty}\left(\mathbb{R}^{+}, H_{0}^{1}(\omega)\right)$ to the unique stationary solution, namely $(M, U[M])$.

Proof. We deduce from Lemma 3.7 that both $\left\|f^{n}(\cdot, \cdot, t)\right\|_{L^{1}(\Omega)}$ and $\left\|f^{n} \log f^{n}(\cdot, \cdot, t)\right\|_{L^{1}(\Omega)}$ are both bounded uniformly in $n \in \mathbb{I N}$ and $t \geq 0$. The stability theorem in [77] implies that up to the extraction of a subsequence $f^{n}$ and $\phi^{n}$ converge weakly in $L^{1}\left(\mathbb{R}_{\mathrm{loc}}^{+} \times \Omega\right)$ and $L^{\infty}\left(\mathbb{R}_{\mathrm{loc}}^{+}, H^{1}(\omega)\right)$ respectively towards a solution of the limit problem. Moreover, since $\lim _{n \rightarrow+\infty} \int_{0}^{T} \Sigma_{\gamma}^{+}\left[f^{n}(\cdot, \cdot, t) \mid M\right] d t=0$, then $\lim _{n \rightarrow+\infty}\left\|f^{n}-M\right\|_{L^{1}([0, T] \times \Gamma)}=0$, which implies

$$
f^{\infty}=M \quad \text { a.e. on } \Gamma .
$$


Besides, the identity

$$
\lim _{n \rightarrow+\infty} \int_{\Omega \times \mathbb{R}^{+}} Q_{\alpha}\left(f^{n}\right) \log \left(\frac{f^{n}}{\left(1-\alpha f^{n}\right) M_{\theta}}\right) d x d v d t=0
$$

implies

$$
\lim _{n \rightarrow+\infty} \int_{\mathbb{R}^{+}}\left(\int_{\Omega}\left|f^{n}\left(1-\alpha f^{n \prime}\right) M_{\theta}-f^{n \prime}\left(1-\alpha f^{n}\right) M_{\theta}^{\prime}\right| d x d v\right)^{2} d t=0 .
$$

On the other hand, due to standard averaging lemmas for kinetic equations [53, 54], we have in the case $\alpha>0$,

$$
f^{n}\left(1-\alpha f^{n \prime}\right) M_{\theta} \rightarrow f^{\infty}\left(1-\alpha f^{\infty \prime}\right) M_{\theta}
$$

weakly in $L^{2}\left(\Omega_{(x, v)} \times \mathbb{R}_{v^{\prime}}^{d} \times[0, T]_{t}\right)$. Therefore we have

$$
f^{\infty}\left(1-\alpha f^{\infty \prime}\right) M_{\theta}=f^{\infty \prime}\left(1-\alpha f^{\infty}\right) M_{\theta}^{\prime} \quad \text { a.e. },
$$

which implies that

$$
Q_{\alpha}\left(f^{\infty}\right)=0 .
$$

We have then proved that $\left(f^{\infty}, \phi^{\infty}\right)$ is a solution of the stationary Vlasov-Poisson system and $f^{\infty}$ is a Fermi-Dirac function. By Lemma 2.3, it is stationary. We deduce from Theorem 2.6 that $f^{\infty}$ is equal to $M$ and there is therefore no need to extract a subsequence.

\subsection{Example 5: Boltzmann or Fokker-Planck-Landau collision operators}

The Boltzmannn equation has been extensively studied during the last 15 years, so we shall only briefly sketch how the case with a Poisson coupling and injection boundary conditions can be dealt with. The main difference with standard approaches is that the total mass is not fixed.

Theorem 3.9 Let $\phi_{0} \in L^{\infty}(\Omega)$ be such that $\nabla \phi_{0} \in W^{1,1}(\Omega)$ and consider a solution $f$ of the VlasovPoisson-Boltzmannn system with a Boltzmann or a Fokker-Planck-Landau collision term such that, with the notations of Section 2,

$$
\Sigma_{\gamma}[f(\cdot, \cdot, t) \mid M] \leq \Sigma_{\gamma}\left[f_{0} \mid M\right] .
$$

Then $t \mapsto \mathcal{M}(t):=\|f(\cdot, \cdot, t)\|_{L^{1}(\Omega)}$ is uniformly bounded in $L^{\infty}\left(\mathbb{R}^{+}\right)$and $\left(f_{n}\right)_{n \in \mathbb{N}}$ defined by (10) weakly converges in $L^{1}\left(\mathbb{R}_{\mathrm{loc}}^{+} \times \Omega\right)$ to $(M, U[M])$.

Note here that the condition on $\phi_{0}$ is certainly not optimal: apart from regularity conditions which have to do with the definition of the characteristics (see $[42,72]$ ), the right condition should be given in terms of the existence of a lower bound for the functional which defines $U[M]$ or equivalently in terms of the existence of a lower bound for $\int_{\Omega} \beta_{\gamma}(f) d x d v$ (see [46] for a discussion of the notion of confinement and $[46,47]$ for the equivalence of these conditions).

Proof. Consider first the case $\alpha>0$ (statistics of fermions) and assume that $\phi_{0} \geq 0$. Since $0 \leq f \leq \frac{1}{\alpha}$ a.e., for almost all $t>0$, the function $f(\cdot, \cdot, t)$ is bounded in $L^{1}(\Omega)$ as soon as $\int_{\Omega} f(x, v, t)|v|^{2} d x d v$ is bounded uniformly with respect to $t$. Let us prove that $f(\cdot, \cdot, t)$ is also relatively compact.

1) for any given $\tau \in(0,1)$ :

$$
\Sigma_{\gamma}[f \mid M] \geq \frac{\tau}{2} \int_{\Omega}|v|^{2} f(x, v, t) d x d v+(1-\tau) \int_{\Omega}\left[f(x, v, t)\left(\frac{1}{2}|v|^{2}+\frac{1}{2} \phi+\phi_{0}\right)+\frac{\theta}{1-\tau} \beta(f)\right] d x d v+C
$$

for some constant $C \in \mathbb{R}$, with $\beta(f)=f \log f+\frac{1}{\alpha}(1-\alpha f) \log (1-\alpha f)$ if $\alpha>0$ and $\beta(f)=f \log f$ if $\alpha=0$. 
2) According to Lemma 3.6, the term

$$
\int_{\Omega}\left[f\left(\frac{1}{2}|v|^{2}+\frac{1}{2} \phi+\phi_{0}\right)+\frac{\theta}{1-\tau} \beta(f)\right] d x d v \geq \frac{\theta}{1-\tau} \int_{\Omega} f \log \left(\frac{f}{g}\right) d x d v
$$

with $\log g=\left(\frac{\theta}{1-\tau}\right)^{-1}\left(\frac{1}{2}|v|^{2}+\frac{1}{2} \phi+\phi_{0}\right)$ is bounded from below by Jensen's inequality:

$$
\int_{\Omega} f \log \left(\frac{f}{g}\right) d x d v \geq\left(\int_{\Omega} f d x d v\right) \log \left(\frac{\int_{\Omega} f d x d v}{\int_{\Omega} g d x d v}\right),
$$

since $g$ is bounded in $L^{1}(\Omega)$.

This also proves that $\int_{\Omega}|v|^{2} f(x, v, t) d x d v$ is uniformly bounded and gives an upper bound for $\mathcal{M}(t)$ :

$$
\Sigma_{\gamma}[f \mid M] \geq \theta \mathcal{M}(t) \log (\mathcal{M}(t))-C \mathcal{M}(t),
$$

for some $C \in \mathbb{R}$. The weak compactness in $L^{1}$ then follows by Dunford-Pettis' criterion (see [50]).

Up to these preliminary estimates, the method is more or less standard and we will only refer to the existing literature. In case $\alpha=0$, for the Boltzmann collision operator, one has to use the notion of renormalized solutions for the Boltzmann equation in $\mathbb{R}^{d}$ (see $[43,44,50]$ or [41] for the Vlasov-Poisson system and an adapted notion of trace [78]). For detailed results, we refer to [79]. As far as we know, there is no satisfactory notion of renormalized solution of the inhomogeneous Fokker-Planck-Landau equation, for which stability results would be available and large time asymptotics only hold at a formal level. Note that relative entropy type estimates are also used in the proof of the existence of stationary solutions (with uncompatible boundary conditions) of the Boltzmann equation in a slab, without Poisson coupling: see [3, 4]. The case of the Boltzmann collision operator with $\alpha>0$ is easier (see [45] for the Cauchy problem in $\mathbb{R}^{d}$ ) and details are left to the reader. Because of the entropy inequality, the limit is therefore in the kernel of $Q_{e e, \alpha}^{B}$, which ends the proof of Theorem 3.9.

\section{Relative entropy and uniqueness}

In this Section, we consider the case without self-consistent potential $\phi$ (no Poisson coupling), when the collision operator is the BGK approximation of the Boltzmann collision operator for fermions given by

$$
Q_{\alpha}(f)=\int_{\mathbb{R}^{d}} \sigma\left(v, v^{\prime}\right)\left[M_{\theta}(v) f\left(v^{\prime}\right)(1-\alpha f(v))-M_{\theta}\left(v^{\prime}\right) f(v)\left(1-\alpha f\left(v^{\prime}\right)\right)\right] d v^{\prime} .
$$

We shall prove by relative entropy methods uniqueness results even when the boundary conditions are not necessarily compatible with $Q_{\alpha}$. The case $\alpha=0$ will be referred as the linear case while the case $\alpha>0$ will be called the nonlinear case. The uniqueness result for stationary solutions (Theorem 2.6) is an extension of the method of Poupaud in [88]. It is a first answer to the long standing open question concerning the uniqueness of the stationary states for nonlinear collision operators like the Boltzmann collision kernel. The method also applies to large time asymptotics (Theorem 2.7).

\subsection{Stationary solutions: proof of Theorem 2.6}

Consider on $\Omega$ the stationary equation

$$
v \cdot \nabla_{x} f-\nabla_{x} \phi_{0} \cdot \nabla_{v} f=Q_{\alpha}(f)
$$

with the boundary condition $f=g$ on $\Gamma^{-}$. Let $f_{1}$ and $f_{2}$ be two solutions. 
The linear case. We assume first that $\alpha=0$ and consider a nonnegative strictly convex function $H: \mathbb{R} \rightarrow \mathbb{R}^{+}$such that $H(0)=0$. Let $h=f_{2}-f_{1}$ and consider $m$ defined by

$$
m(x, v)=e^{-\left(\frac{1}{2}|v|^{2}+\phi_{0}(x)\right) / \theta} \quad \forall(x, v) \in \Omega .
$$

It is a stationary solution of the Vlasov equation

$$
v \cdot \nabla_{x} m-\nabla_{x} \phi_{0} \cdot \nabla_{v} m=0
$$

(which does not satisfy the injection condition on $\Gamma^{-}$). The function $m H\left(\frac{h}{m}\right)$ satisfies

$$
\left(v \nabla_{x}-\nabla_{x} \phi_{0} \cdot \nabla_{v}\right)\left(m H\left(\frac{h}{m}\right)\right)=Q_{0}(h) H^{\prime}\left(\frac{h}{m}\right) .
$$

Integrating this identity with respect to $x$ and $v$ leads to

$$
\int_{\Gamma^{+}} m H\left(\frac{h}{m}\right) d \sigma-\int_{\Omega} Q_{0}(h) H^{\prime}\left(\frac{h}{m}\right) d x d v=0 .
$$

Since $H$ is positive on $\mathbb{R} \backslash\{0\}$ and $H^{\prime}$ is strictly increasing on $\mathbb{R}^{+}$, both terms have to vanish (for any fixed $x \in \omega, m(x, v)$ is proportional to $\left.m_{\theta}(v)\right)$. Therefore $h$ vanishes on the boundary $\partial \Omega$ and $Q_{0}(h)=0$, which is possible only if $\frac{h^{\prime}}{m_{\theta}^{\prime}}=\frac{h}{m_{\theta}}$ a.e. in $\Omega$ : there is a function $\rho(x)$ defined on $\Omega$ such that $h(x, v)=\rho(x) m_{\theta}(v)$ for any $(x, v) \in \Omega$. Since $h$ is a solution of (13), we immediately obtain $\nabla_{x} \rho-\rho \nabla_{x} \phi_{0}=0: \rho \equiv 0=h_{\mid \Gamma}$.

The nonlinear case. The proof in this case is slightly more complicated. It relies on the use of the inequality

$$
\int_{\mathbb{R}^{d}}\left[Q_{\alpha}\left(f_{1}\right)-Q_{\alpha}\left(f_{2}\right)\right] \cdot \operatorname{sgn}\left(f_{1}-f_{2}\right) d v \leq 0
$$

with equality if and only if $f_{1}-f_{2}$ has a constant sign. This is a consequence of the identity

$\int_{\mathbb{R}^{d}}\left[Q_{\alpha}\left(f_{1}\right)-Q_{\alpha}\left(f_{2}\right)\right] \cdot \operatorname{sgn}\left(f_{1}-f_{2}\right) d v=-\frac{1}{2} \int_{\mathbb{R}^{d}} \sigma\left(v, v^{\prime}\right)\left|f_{1}-f_{2}\right|\left(1-\operatorname{sgn}\left(f_{1}-f_{2}\right) \cdot \operatorname{sgn}\left(f_{1}^{\prime}-f_{2}^{\prime}\right)\right) \cdot P d v d v^{\prime}$,

where certainly $P:=M_{\theta}^{\prime}\left(1-\alpha f_{1}^{\prime}\right)+\alpha M_{\theta} f_{2}^{\prime}+M_{\theta}^{\prime}\left(1-\alpha f_{2}^{\prime}\right)+\alpha M_{\theta} f_{1}^{\prime}$ is positive a.e. and $1-\operatorname{sgn}\left(f_{1}-\right.$ $\left.f_{2}\right) \cdot \operatorname{sgn}\left(f_{1}^{\prime}-f_{2}^{\prime}\right) \geq 0$. We proceed in analogy with the linear case. Namely, if we consider two solutions $f_{1}$ and $f_{2}$ of (13), the difference $h=f_{1}-f_{2}$ satisfies

$$
v \cdot \nabla_{x}|h|-\nabla_{x} \phi_{0} \cdot \nabla_{v}|h|=\left[Q_{\alpha}\left(f_{1}\right)-Q_{\alpha}\left(f_{2}\right)\right] \cdot \operatorname{sgn}(h) .
$$

with zero inflow boundary conditions. Integrating this equation with respect to $x$ and $v$ yields

$$
\int_{\Gamma^{+}}|h| d \sigma-\int_{\Omega}\left[Q_{\alpha}\left(f_{1}\right)-Q_{\alpha}\left(f_{2}\right)\right] \cdot \operatorname{sgn}(h) d x d v=0
$$

which implies that $h$ vanishes on the whole boundary $\partial \Omega$ and that for almost every $x \in \Omega, h(x, \cdot)$ has a constant sign (which may depend on $x$ ). The last property can be rewritten as follows: for any function $S\left(x, v, v^{\prime}\right)$ for which the integrals make sense,

$$
\left[\int S\left(x, v, v^{\prime}\right) h\left(x, v^{\prime}\right) d v^{\prime}\right] \cdot \operatorname{sgn}(h(x, v))=\int S\left(x, v, v^{\prime}\right)\left|h\left(x, v^{\prime}\right)\right| d v^{\prime} .
$$

Thus $h$ is a solution of

$$
v \cdot \nabla_{x}|h|-\nabla_{x} \phi_{0} \cdot \nabla_{v}|h|+\lambda|h|=\int_{\mathbb{R}^{d}} \sigma_{1}\left|h^{\prime}\right| d v^{\prime}
$$


with $h=0$ on $\partial \Omega$,

$$
\begin{aligned}
& \lambda(x, v)=\int_{\mathbb{R}^{d}} \sigma\left(v, v^{\prime}\right)\left[\alpha M_{\theta}(v) f_{1}\left(x, v^{\prime}\right)+\left(1-\alpha f_{1}\left(x, v^{\prime}\right)\right) M_{\theta}\left(v^{\prime}\right)\right] d v^{\prime} \\
& \text { and } \sigma_{1}\left(x, v, v^{\prime}\right)=\sigma\left(v, v^{\prime}\right)\left[M_{\theta}(v)\left(1-\alpha f_{2}(x, v)\right)+\alpha M_{\theta}\left(v^{\prime}\right) f_{2}(x, v)\right]
\end{aligned}
$$

The function $\lambda$ is a bounded positive function. We claim that this implies that $h=0$. Indeed, let $\left(x_{0}, v_{0}\right) \in \Omega$ with $\left|v_{0}\right|$ large enough in such a way that any characteristics with initial conditions in $(x, v) \in B_{r}\left(x_{0}\right) \times B_{r}\left(v_{0}\right)$ (with $r>0$ ) is open. Let $\psi$ be a nonnegative smooth function which is strictly positive on $B_{r}\left(x_{0}\right) \times B_{r}\left(v_{0}\right)$. The solution $\theta$ of

$$
v \cdot \nabla_{x} \theta-\nabla_{x} \phi_{0} \cdot \nabla_{v} \theta-\lambda \theta=\psi, \quad \theta=0 \text { on } \Sigma^{-},
$$

is nonnegative and does not vanish on $B_{r}\left(x_{0}\right) \times B_{r}\left(v_{0}\right)$. Let us give a short proof of this fact. Assume that the characteristics which is given by

$$
\begin{array}{ll}
\frac{\partial X}{\partial t}=V, & \frac{\partial V}{\partial t}=-\nabla_{x} \phi_{0}(X), \\
X(x, v, 0)=x, & V(x, v, 0)=v,
\end{array}
$$

exists on a maximal interval $\left(\mathcal{T}_{i n}(x, v), \mathcal{T}_{e}(x, v)\right) \ni 0$. If such a characteristics is open, this means that either $\mathcal{T}_{\text {in }}(x, v)>-\infty$ and $X\left(x, v, \mathcal{T}_{\text {in }}(x, v)\right) \in \partial \omega$, or $\mathcal{T}_{e}(x, v)<+\infty$ and $X\left(x, v, \mathcal{T}_{e}(x, v)\right) \in \partial \omega$. Note that since $\theta$ is a steady state, the problem is autonomous, so we dont need to introduce a specific initial time (with the notations of $(12), X(t ; x, v, s)$ is replaced by $X(t-s ; x, v, 0)=X(x, v, t-s)$ ). Let $\Lambda(x, v, t)=\int_{0}^{t} \lambda(X(s), V(s)) d s$. If $\mathcal{T}_{\text {in }}(x, v)>-\infty, \theta$ is represented by the integral formula

$$
\theta(x, v)=\int_{\mathcal{T}_{\text {in }}(x, v)}^{0} e^{-\Lambda(x, v, s)} \psi(X(s), V(s)) d s,
$$

is therefore nonnegative and does not vanish on $B_{r}\left(x_{0}\right) \times B_{r}\left(v_{0}\right)$. A similar formula holds if $\mathcal{T}_{e}<+\infty$.

Using (16), (17) and Green's formula, we deduce that

$$
\int_{\Omega}|h(x, v)| \psi(x, v)+\int_{\Omega}\left(\int_{\mathbb{R}^{d}} \sigma_{1}\left(x, v, v^{\prime}\right)\left|h\left(x, v^{\prime}\right)\right| d v^{\prime}\right) \theta(x, v) d x d v=0 .
$$

which implies that $\int_{\mathbb{R}^{d}} \sigma_{1}\left(x, v, v^{\prime}\right)\left|h\left(x, v^{\prime}\right)\right| d v^{\prime}$ vanishes on $B_{r}\left(x_{0}\right) \times B_{r}\left(v_{0}\right)$. Since $\sigma_{1}$ does not vanish, this implies that $h$ vanishes on $B_{r}\left(x_{0}\right) \times \mathbb{R}^{d}$. We conclude that $h$ vanishes identically, since $x_{0}$ can be arbitrarily chosen in $\omega$.

\subsection{Large time asymptotics: proof of Theorem 2.7}

The above method is also usefull for the study of large time asymptotics. It gives the convergence to the unique stationary solution and shows the connection with relative entropy formulations which have been extensively used throughout the rest of this paper. We denote by $h$ the function $f-f_{s}$.

The linear case. Multiplying the Equation (15) by $H^{\prime}\left(\frac{h}{m}\right)$ and integrating with respect to $(x, v)$, we obtain the identity

$$
\frac{d}{d t} \int_{\Omega} m H\left(\frac{h}{m}\right) d x d v+\int_{\Gamma^{+}} m H\left(\frac{h}{m}\right) d \sigma-\int_{\Omega} Q_{0}(h) H^{\prime}\left(\frac{h}{m}\right) d x d v=0 .
$$

According to the same strategy as in Section 3 , we define $h_{n}(x, v, t)=h\left(t+t_{n}, x, v\right)$ where $t_{n}$ is an arbitrary diverging sequence and deduce that up to the extraction of a subsequence, the sequence $h_{n}$ *-weakly converges in $L^{\infty}((0, T) \times \Omega)$ towards a function $h_{\infty}$ such that $Q_{0}\left(h_{\infty}\right)=0, \partial_{t} h_{\infty}+v \cdot \nabla_{x} h_{\infty}-$ 
$\nabla_{x} \phi_{0} \cdot \nabla_{v} h_{\infty}=0$ and $h_{\infty}=0$ on $\partial \Omega$. The first identity implies that $h_{\infty}$ is a Maxwellian, which is even with respect to $v$ and is therefore stationary in view of Lemma 2.3. Theorem 2.6 then implies that $h_{\infty}=0$.

The nonlinear case. As in the proof of Theorem 2.6, a simple computation gives

$$
\frac{d}{d t} \int_{\Omega}|h| d x d v+\int_{\Gamma^{+}}|h| d \sigma-\int_{\Omega}\left[Q_{\alpha}(f)-Q_{\alpha}\left(f_{s}\right)\right] \cdot \operatorname{sgn}(h) d x d v=0 .
$$

The $*$-weak limit $h_{\infty}^{+}$in $L^{\infty}((0, T) \times \Omega)$ of a subsequence of $h_{n}^{+}=\left|h\left(\cdot, \cdot, \cdot+t_{n}\right)\right|$ defined as above satisfies

$$
\begin{gathered}
\partial_{t} h_{\infty}^{+}+v \cdot \nabla_{x} h_{\infty}^{+}-\nabla_{x} \phi_{0} \cdot \nabla_{v} h_{\infty}^{+}+\lambda_{\infty} h_{\infty}^{+}=\int_{\mathbb{R}^{d}} \sigma_{1}\left|\left(h_{\infty}^{+}\right)^{\prime}\right| d v^{\prime} \\
\text { where } \quad \lambda_{\infty}=\int_{\mathbb{R}^{d}} \sigma\left(v, v^{\prime}\right)\left[\alpha M_{\theta}(v) f_{\infty}\left(x, v^{\prime}\right)+\left(1-\alpha f_{\infty}\left(x, v^{\prime}\right)\right) M_{\theta}\left(v^{\prime}\right)\right] d v^{\prime} \\
\text { and } \sigma_{1}\left(x, v, v^{\prime}\right)=\sigma\left(v, v^{\prime}\right)\left[M_{\theta}(v)\left(1-\alpha f_{s}(x, v)\right)+\alpha M_{\theta}\left(v^{\prime}\right) f_{s}(x, v)\right]
\end{gathered}
$$

where $f_{\infty}$ is, up to the extraction of a further subsequence, the limit of $f\left(\cdot, \cdot, \cdot+t_{n}\right)$. The convergence in the collision term holds for the same reason as in the proof of Theorem 2.6. On the other hand $h_{\infty}^{+}(x, v, t)=0$ for all $t>0,(x, v) \in \partial \Omega$. As in the proof of Theorem 2.6 again, this implies that $h_{\infty}^{+}=0$. But since $\left|f_{\infty}-f_{s}\right| \leq h_{\infty}^{+}$, we deduce that $f_{\infty}=f_{s}$.

\section{$5 \quad$ Other boundary conditions}

This section is devoted to further considerations on relative entropies corresponding to various types of boundary conditions. The case of diffuse reflection boundary conditions is studied with some details: after a definition of such boundary conditions, which are such that the total mass is preserved, stationary solutions are found using a variational approach. These solutions are then used to define a relative entropy, which describes the irreversibility, and gives the uniqueness of the stationary solution when there is no closed characteristics, exactly like in the case of injection boundary conditions. Conditions preserving the energy and the mass are then introduced and further remarks are done concerning other types of possible boundary conditions.

\subsection{Diffuse reflection boundary conditions (DRBC)}

Here we consider as in Section 1 the full Vlasov-Poisson-Boltzmann system

$$
\left\{\begin{array}{l}
\partial_{t} f+v \cdot \nabla_{x} f-\left(\nabla_{x} \phi+\nabla_{x} \phi_{0}\right) \cdot \nabla_{v} f=Q(f) \\
-\Delta \phi=\int_{\mathbb{R}^{d}} f(x, v, t) d v \\
\phi_{\mid \partial \omega}=0
\end{array}\right.
$$

and diffuse reflection boundary conditions for $f$. These conditions are defined as follows. For any $(x, t) \in \partial \omega \times \mathbb{R}^{+}$, let

$$
\rho_{+}(x, t):=\int_{\Sigma^{+}(x)} f(x, v, t) v \cdot \nu(x) d v .
$$

Assuming that $\gamma$ is defined on $\mathbb{R}$, satisfies $(\mathcal{P})$ and is such that $\lim _{s \rightarrow-\infty} \gamma(s)=+\infty$, there exists a unique function $\mu: \partial \omega \times \mathbb{R}^{+} \rightarrow \mathbb{R}$ for which

$$
\rho_{+}(x, t)=\int_{\sum^{-}(x)} \gamma\left(\frac{1}{2}|v|^{2}+\phi_{0}(x)-\mu(x, t)\right)|v \cdot \nu(x)| d v \quad \forall(x, t) \in \partial \omega \times \mathbb{R}^{+} .
$$


With the notation

$$
m_{f}(x, v, t):=\gamma\left(\frac{1}{2}|v|^{2}+\phi_{0}(x)-\mu(x, t)\right) \quad \forall(x, t) \in \partial \omega \times \mathbb{R}^{d} \times \mathbb{R}^{+},
$$

we shall say that $f$ is subject to diffuse reflection boundary conditions (DRBC) if and only if

$$
f(x, v, t)=m_{f}(x, v, t), \quad \forall t \in \mathbb{R}^{+}, \quad \forall(x, v) \in \Gamma^{-} .
$$

Note that under this condition, the total mass is preserved:

$$
\frac{d}{d t} \int_{\Omega} f(x, v, t) d x d v=-\int_{\Omega} \operatorname{div}_{x}(v f(x, v, t)) d x d v=-\int_{\Gamma} f(x, v, t) d \tilde{\sigma}(x, v)=0,
$$

where $d \tilde{\sigma}(x, v)=v \cdot \nu(x) d_{\partial \omega}(x) \cdot d v$ is a signed measure such that $d \tilde{\sigma}= \pm d \sigma$ on $\Gamma^{ \pm}$, with the notations of Section 1. From now on, we denote by $\mathcal{M}$ the $L^{1}$-norm of $f$ :

$$
\mathcal{M}=\|f(\cdot, \cdot, t)\|_{L^{1}(\Omega)} \quad \forall t \in \mathbb{R}^{+} .
$$

Under DRBC conditions, we shall now prove the existence of a stationary solution corresponding to any given mass by the mean of a variational approach. This solution then allows us to define a relative entropy, which we shall use to prove the uniqueness of the stationary solution. This relative entropy also describes the irreversibility and the large time asymptotics as in the case of injection boundary conditions. See the concluding remark of this section for further comments on the denomination: relative.

\subsubsection{A variational formulation in the (DRBC) case}

Lemma 5.1 Assume that $\phi_{0}$ is a bounded from below measurable function. For any $\mathcal{M}>0$, system (18) supplemented with boundary conditions zero Dirichlet boundary conditions for $\phi$ and (21) for $f$ has at least one nonnegative stationary solution $M$ such that $\|M\|_{L^{1}(\Omega)}=\mathcal{M}$.

Proof. We look for a solution $M$ satisfying the fixed-point equation

$$
M(x, v)=\gamma\left(\frac{1}{2}|v|^{2}+\phi_{0}(x)+U[M]-\mu_{\mathcal{M}}\right),
$$

where $\mu_{\mathcal{M}}$ is a constant such that

$$
\int_{\Omega} \gamma\left(\frac{1}{2}|v|^{2}+\phi_{0}(x)+U[M]-\mu_{\mathcal{M}}\right) d x d v=\mathcal{M}
$$

(this constant is unique at least for $U[M]$ fixed). For such an $M$, the Vlasov equation in (18) is certainly satisfied. With the same notations as in Section 1, if

$$
g(u)=\int_{\mathbb{R}^{d}} \gamma\left(\frac{1}{2}|v|^{2}+u\right) d v=2^{d / 2-1}\left|S^{d-1}\right| \int_{0}^{+\infty} s^{d / 2-1} \gamma(s+u) d s,
$$

to solve the Poisson equation in (18) with $f \equiv M$ given by (22) is therefore equivalent to solve

$$
-\Delta \phi=g\left(\phi+\phi_{0}-\mu\left[\phi+\phi_{0}\right]\right)
$$

where $\mu: W_{0}^{1,(d-1) / d}(\omega) \rightarrow \mathbb{R}$ is a functional implicitly defined by the condition

$$
\int_{\omega} g(u-\mu[u]) d x=\mathcal{M}
$$


We refer to [47] for a complete resolution of (24) in a similar case (Neumann boundary conditions). Let us give a sketch of the proof. The function $\phi$ is a critical point in $H_{0}^{1} \cap W^{1,(d-1) / d}(\omega)$ of

$$
J[\phi]=\frac{1}{2} \int_{\omega}|\nabla \phi|^{2} d x-\int_{\omega} G\left(\phi+\phi_{0}-\mu\left[\phi+\phi_{0}\right]\right) d x+\mathcal{M} \mu\left[\phi+\phi_{0}\right],
$$

where $G$ is a primitive of $g$. This functional is convex as can be checked by direct investigation. It is actually easier to minimize

$$
H[f]=\int_{\Omega}\left(\beta_{\gamma}(f)+f\left(\frac{1}{2}|v|^{2}+\phi_{0}\right)\right) d x d v+\frac{1}{2} \int_{\omega}|\nabla U[f]|^{2} d x
$$

on $\left\{f \in L_{+}^{1}(\Omega): \int_{\Omega} f d x d v=\mathcal{M}\right\}$, where $\beta_{\gamma}(f)=-\int_{0}^{f} \gamma^{-1}(z) d z . H$ is also convex ( $H$ and $J$ are related in terms of convex conjugation by Legendre's transform: see [47]), and a critical point $f$ of $H$ has to satisfy:

$$
-\gamma^{-1}(f)+U[f]+\phi_{0}-\lambda=0,
$$

where $\lambda=\mu\left[U[f]+\phi_{0}\right]=\mu_{\mathcal{M}}$ (according to (23), since $f \equiv M$ by the above equation) is the Lagrange multiplier associated to the constraint $\int_{\Omega} f d x d v=\mathcal{M}$ : it is straightforward to check that such a critical point $f$ exists, which immediately gives a solution $M=f$ to Equation (22). It is moreover clear that the boundary condition (21) has to be satisfied, with $\mu(x, t) \equiv \mu_{\mathcal{M}}$.

At this point, we notice that the solution (22) of (24) is unique because of the convexity of $H$, but we did not prove yet that any stationary solution of (18) is necessarily of the form (22).

\subsubsection{Relative entropy in the (DRBC) case}

Like in the case of injection boundary conditions, we may define the relative entropy and the boundary entropy flux respectively by

$$
\begin{aligned}
& \Sigma_{\gamma}[g \mid h]=\int_{\Omega}\left(\beta_{\gamma}(g)-\beta_{\gamma}(h)-(g-h) \beta_{\gamma}^{\prime}(h)\right) d x d v+\frac{1}{2} \int_{\omega}|\nabla U[g-h]|^{2} d x \\
& \text { and } \Sigma_{\gamma}^{+}[g \mid h]=\int_{\Gamma^{+}}\left(\beta_{\gamma}(g)-\beta_{\gamma}(h)-(g-h) \beta_{\gamma}^{\prime}(h)\right) d \sigma .
\end{aligned}
$$

Recall that $\Sigma_{\gamma}[g \mid h]$ and $\Sigma_{\gamma}^{+}[g \mid h]$ are always nonnegative and vanish if and only if $g=h$ a.e. on $\Omega$ and $\Gamma^{+}$respectively. Exactly as in the injection boundary conditions case, we may first state a result on the irreversibility.

Theorem 5.2 Let $(f, \phi)$ be a solution of (18) supplemented with diffuse reflection (21) and zero Dirichlet boundary conditions respectively, $\mathcal{M}=\|f(\cdot, \cdot, 0)\|_{L^{1}(\Omega)}$ and assume that Assumptions (H1)(H4) hold. We further assume that $\gamma$ is defined on $\mathbb{R}$ and $\lim _{s \rightarrow-\infty} \gamma(s)=+\infty$. Then the relative entropy satisfies

$$
\frac{d}{d t} \Sigma_{\gamma}[f(t) \mid M]=-\Sigma_{\gamma}^{+}\left[f \mid m_{f}\right]+\int_{\Omega} Q(f)\left[\frac{1}{2}|v|^{2}-\gamma^{-1}(f)\right] d x d v \leq 0,
$$

where $M$ is defined by (22)-(23).

Proof. The computations are similar to the case of injection boundary conditions. Notice first that

$$
-\int_{\Omega}(f-M) \beta_{\gamma}^{\prime}(M) d x d v=\int_{\Omega}(f-M)\left(\frac{1}{2}|v|^{2}+\phi_{0}(x)+U[M]-\mu_{\mathcal{M}}\right) d x d v .
$$

Because of the conservation of mass and (23), $\mu_{\mathcal{M}} \int_{\Omega}(f-M) d x d v=0$, and using the properties of $U$,

$$
\int_{\Omega}(f-M) U[M] d x d v+\frac{1}{2} \int_{\omega}|\nabla U[f-M]|^{2} d x=\frac{1}{2} \int_{\omega}\left(|\nabla U[f]|^{2}-|\nabla U[M]|^{2}\right) d x .
$$


Thus, only the collision term and the boundary terms contribute to $\frac{d}{d t} \Sigma_{\gamma}[f(t) \mid M]$ :

$$
\begin{aligned}
& \frac{d}{d t} \Sigma_{\gamma}[f(t) \mid M]-\int_{\Omega} Q(f)\left[\frac{1}{2}|v|^{2}-\gamma^{-1}(f)\right] d x d v \\
& \quad=\int_{\Gamma}\left(\beta_{\gamma}(f)-\beta_{\gamma}(M)-(f-M)\left(\frac{1}{2}|v|^{2}+\phi_{0}(x)-\mu_{\mathcal{M}}\right)\right) d \tilde{\sigma}(x, v) .
\end{aligned}
$$

Certainly,

$$
\int_{\Gamma}\left(\beta_{\gamma}(M)-M\left(\frac{1}{2}|v|^{2}+\phi_{0}(x)-\mu_{\mathcal{M}}\right)\right) d \tilde{\sigma}(x, v)=0
$$

but also

$$
\int_{\Gamma}\left(\beta_{\gamma}\left(m_{f}\right)-m_{f}\left(\frac{1}{2}|v|^{2}+\phi_{0}(x)-\mu(x, t)\right)\right) d \tilde{\sigma}(x, v)=0,
$$

by definition (20) of $\mu(x, t)$. According to (19) and the boundary condition (21), we get $\int_{\Gamma} f d \tilde{\sigma}(x, v)=0$ and $\int_{\Gamma} f(x, v, t) \mu(x, t) v \cdot \nu(x) d v=0$. Thus

$$
\begin{aligned}
& \int_{\Gamma}\left(\beta_{\gamma}(f)-f\left(\frac{1}{2}|v|^{2}+\phi_{0}(x)-\mu_{\mathcal{M}}\right)\right) d \tilde{\sigma}(x, v) \\
& \quad=\int_{\Gamma}\left(\beta_{\gamma}(f)-f\left(\frac{1}{2}|v|^{2}+\phi_{0}(x)-\mu(x, t)\right)\right) d \tilde{\sigma}(x, v) \\
& \quad=\int_{\Gamma^{+}}\left(\beta_{\gamma}(f)-\beta_{\gamma}\left(m_{f}\right)-\beta_{\gamma}^{\prime}\left(m_{f}\right)\left(f-m_{f}\right)\right) d \sigma(x, v)
\end{aligned}
$$

Since $\beta_{\gamma}^{\prime}\left(m_{f}(x, v, t)\right)=\frac{1}{2}|v|^{2}+\phi_{0}(x)-\mu(x, t)$, Theorem 5.2 is proved.

Corollary 5.3 Under the same assumptions as in Theorem 5.2, if there are no closed trajectories, and if $\omega$ is a $C^{1}$ bounded connected domain in $\mathbb{R}^{d}$, then for any $\mathcal{M}>0$, there exists a unique continuous nonnegative stationary solution of (18)-(21) with $L^{1}$-norm $\mathcal{M}$. This solution, $M$, is given by (22)-(23).

The condition on $\phi_{0}$ for the uniqueness of the stationary solution (no closed trajectories) is exactly the same as for the injection boundary conditions. For instance, in dimension $d=1$, it would be sufficient to assume that $-\phi_{0}^{\prime \prime}>0$.

Proof. Because of Theorem 5.2, if $f$ is a solution of (18)-(21), then

$$
f(x, v)=m_{f}(x, v)=\gamma\left(\frac{1}{2}|v|^{2}+\phi_{0}(x)-\mu(x)\right), \quad \forall(x, v) \in \Gamma
$$

(here we omit the dependence of $\mu$ in $t$ since $f$ does not depend on $t$ either). In the following, we shall do as if $\phi$ and $\phi_{0}$ were of class $C^{2}$. For lower regularity, one has to take advantage of the uniqueness of the characteristics according to [42]. Consider two points $x_{1}$ and $x_{2}$ in $\partial \omega$ such that the segment $\left(x_{1}, x_{2}\right)$ is a subset of $\omega$ : there exists a characteristics connecting $x_{1}$ to $x_{2}$ (Bolza problem in $\mathbb{R}^{d}$ : see [95] and Appendix B), i.e. a solution of

$$
\frac{d X}{d t}=V, \quad \frac{d V}{d t}=-\nabla\left(\phi(X)+\phi_{0}(X)\right), \quad X(0)=x_{1}, \quad V(0)=v_{1}
$$

such that for some $t>0, x(t)=x_{2}$, for some well chosen $v_{1}$, with $\left|v_{1}\right|$ large enough. Since $f$ is constant along the characteristics, $f\left(x_{2}, v_{2}\right)=f\left(x_{1}, v_{1}\right)$. Because $f$ only depends on the energy on the boundary (up to $\mu$ ) and since $\gamma$ is strictly decreasing, we have:

$$
\frac{1}{2}\left|v_{2}\right|^{2}+\phi_{0}\left(x_{2}\right)-\mu\left(x_{2}\right)=\frac{1}{2}\left|v_{1}\right|^{2}+\phi_{0}\left(x_{1}\right)-\mu\left(x_{1}\right) .
$$

But on the other hand, $\phi$ does not depend on $t$ and the energy also is conserved along the characteristics:

$$
\frac{1}{2}\left|v_{2}\right|^{2}+\phi_{0}\left(x_{2}\right)=\frac{1}{2}\left|v_{1}\right|^{2}+\phi_{0}\left(x_{1}\right) .
$$


This is possible if and only if $\mu\left(x_{2}\right)=\mu\left(x_{1}\right)$. See Lemma B.1 in Appendix B for more details on how to find $v$. It remains to check that any two points of a $C^{1}$ connected domain in $\mathbb{R}^{d}$ can be connected by a finite number of segments in $\omega$, whose extremities are in $\partial \omega$. This is the purpose of Lemma B.2 in Appendix B. Thus $\mu(x)$ defined by (19) does not depend on $x$ and we can conclude by applying Lemma 5.1.

Depending on the form of the collision kernel, large time results similar to the ones corresponding to injection boundary conditions of Sections 1-4 can also be established. The adaptation of the proofs is left to the reader.

\subsection{Diffuse reflection boundary conditions preserving the energy}

Consider a solution of (18). For any $(x, t) \in \partial \omega \times \mathbb{R}^{+}$, consider

$$
e_{+}(x, t):=\int_{\Sigma^{+}(x)}\left(\frac{1}{2}|v|^{2}+\phi_{0}(x)\right) f(x, v, t) v \cdot \nu(x) d v
$$

and, as in the case without preservation of the energy,

$$
\rho_{+}(x, t):=\int_{\Sigma^{+}(x)} f(x, v, t) v \cdot \nu(x) d v .
$$

Because of the monotonicity properties of $\gamma$, there exist two functions $\mu, T: \partial \omega \times \mathbb{R}^{+} \rightarrow \mathbb{R}$ such that

$$
\begin{gathered}
\rho_{+}(x, t)=\int_{\Sigma^{+}(x)} \gamma\left(\frac{1}{T(x, t)}\left[\frac{1}{2}|v|^{2}+\phi_{0}(x)-\mu(x, t)\right]\right) v \cdot \nu(x) d v \\
e_{+}(x, t)=\int_{\Sigma^{+}(x)}\left(\frac{1}{2}|v|^{2}+\phi_{0}(x)\right) \gamma\left(\frac{1}{T(x, t)}\left[\frac{1}{2}|v|^{2}+\phi_{0}(x)-\mu(x, t)\right]\right) v \cdot \nu(x) d v .
\end{gathered}
$$

With the notation

$$
m_{f}(x, v, t):=\gamma\left(\frac{1}{T(x, t)}\left[\frac{1}{2}|v|^{2}+\phi_{0}(x)-\mu(x, t)\right]\right),
$$

for any $(x, v, t) \in \partial \omega \times \mathbb{R}^{d} \times \mathbb{R}^{+}, f$ is subject to diffuse reflection boundary conditions preserving the energy if and only if

$$
f(x, v, t)=m_{f}(x, v, t), \quad \forall t \in \mathbb{R}^{+}, \quad \forall(x, v) \in \Gamma^{-} .
$$

Under these boundary conditions, the mass $\|f(\cdot, \cdot, t)\|_{L^{1}(\Omega)}=\mathcal{M}$ and the total energy

$$
\mathcal{E}=\int_{\Omega}\left(\frac{1}{2}|v|^{2}+\phi_{0}(x)+\frac{1}{2} U[f]\right) f(x, v, t) d x d v
$$

are conserved. We may then consider a stationary state taking the form

$$
M(x, v)=\gamma\left(\frac{1}{T_{\mathcal{E}, \mathcal{M}}}\left[\frac{1}{2}|v|^{2}+\phi_{0}(x)+U[M]-\mu_{\mathcal{E}, \mathcal{M}}\right]\right),
$$

where $\mu_{\mathcal{E}, \mathcal{M}}, T_{\mathcal{E}, \mathcal{M}}$ are constants such that

$$
\begin{aligned}
& \int_{\Omega} \gamma\left(\frac{1}{T_{\mathcal{E}, \mathcal{M}}}\left[\frac{1}{2}|v|^{2}+\phi_{0}(x)+U[M]-\mu_{\mathcal{E}, \mathcal{M}}\right]\right) d x d v=\mathcal{M} \\
& \text { and } \quad \int_{\Omega}\left(\frac{1}{2}|v|^{2}+\phi_{0}(x)+\frac{1}{2} U[f]\right) \gamma\left(\frac{1}{T_{\mathcal{E}, \mathcal{M}}}\left[\frac{1}{2}|v|^{2}+\phi_{0}(x)+U[M]-\mu_{\mathcal{E}, \mathcal{M}}\right]\right) d x d v=\mathcal{E} .
\end{aligned}
$$


The existence of such a stationary solution can be proved by variational arguments, but whether uniqueness holds or not is in general open (see [17]). Note that because of the conservation of mass and energy,

$$
\left.\frac{d}{d t}\left[\int_{\Omega}(f-M) \beta_{\gamma}^{\prime}(M)\right) d x d v+\frac{1}{2 T_{\mathcal{E}, \mathcal{M}}} \int_{\omega}|\nabla U[f-M]|^{2} d x\right]=0,
$$

and the relative entropy takes the simplified form

$$
\Sigma_{\gamma}[f \mid M]=\int_{\Omega}\left(\beta_{\gamma}(f)-\beta_{\gamma}(M)\right) d x d v .
$$

The irreversibility is measured by this relative entropy (which coincides with the usual notion of entropy in thermodynamics, up to a sign convention). Let $\Sigma_{\gamma}^{+}\left[f \mid m_{f}\right]=\int_{\Gamma^{+}}\left(\beta_{\gamma}(f)-\beta_{\gamma}\left(m_{f}\right)\right) d \sigma$.

Proposition 5.4 With the above notations,

$$
\frac{d}{d t} \Sigma_{\gamma}[f(t) \mid M]=-\Sigma_{\gamma}^{+}\left[f \mid m_{f}\right]+\int_{\Omega} Q(f)\left[\frac{1}{2}|v|^{2}-\gamma^{-1}(f)\right] d x d v \leq 0 .
$$

By an argument similar to the one of Corollary 5.3, it is then easy to prove that any stationary solution is necessarily of the form (25).

\subsection{Remarks on the boundary conditions}

To the boundary conditions for $f$ correspond various well known situations of thermodynamics (see [8]). In the case of injection (resp. diffuse reflection) boundary conditions, the temperature and the chemical potential (resp. the temperature and the mass) are fixed, so that the energy and the mass (resp. the energy and the chemical potential) of the system fluctuate: this is the grand canonical (resp. canonical) framework and the relative entropy can be identified with a grand potential (resp. free energy) function. The stationary state is uniquely defined in both cases.

When the energy and the mass are fixed (microcanonical framework), the relative entropy can be identified with an entropy function (in the usual sense of thermodynamics, up to a sign convention), but a difficulty arises from the lack of uniqueness results of stationary states (see $[38,17]$ ). Other cases formally enter in our relative entropy formulation: for instance, if the volume is not fixed, one could prescribe the pressure by requiring the equality of the incoming and outgoing fluxes corresponding to the first moment in the velocity.

Remark 5.5 Why we use the denomination relative for the entropy arises from the following reason. In the three examples of boundary conditions studied in this paper (injection boundary conditions, diffuse reflection boundary conditions with fixed temperature and diffuse reflection boundary conditions preserving mass and energy), the function $\beta_{\gamma}$ is entirely defined by $\gamma$, but we further impose that the minimum of $\Sigma_{\gamma}$ is reached by the unique stationary solution corresponding to the boundary conditions. This in turn determines the Lagrange multipliers associated to the constraints. In that sense, the entropy is therefore relative to this stationary solution. The relative entropy functional can be interpreted - from a probabilistic point of view - as a conditional expectation, or simply as a measure of the distance to the stationary state (at least when it is unique). This notion of distance is also the one which appears when measuring the stability by the Casimir-energy method or in case of diffusion equations with compatible nonlinearities (see [27, 18]), as we shall see in Appendices $C$ and D.

\section{A End of the proof of Theorem 2.5}

Let $\Phi:[0,1] \times \mathbb{R} \rightarrow \mathbb{R},(x, t) \mapsto \Phi(x, t)$ be an analytic function in $x$ with $C^{\infty}$ in time coefficients. Assume that:

- $\Phi(0, t)=0$ and $\Phi(1, t)=\Phi_{1}$ do not depend on $t$, 
- $\frac{\partial \Phi}{\partial x}(0, t) \geq C_{2}>0$ uniformly in $t \in \mathbb{R}$.

We define the characteristics $(X, V)(t ; x, v, s)$ as the solution of

$$
\begin{array}{ll}
\frac{\partial X}{\partial t}=V, & \frac{\partial V}{\partial t}=-\frac{\partial \Phi}{\partial x}(X, t), \\
X(t ; x, v, t)=x, & V(t ; x, v, t)=v,
\end{array}
$$

and assume that

$$
\left|V_{i n}\left(t_{0}, x_{0}, 0\right)\right|=\left|V_{e}\left(t_{0}, x_{0}, 0\right)\right|, \quad \forall\left(x_{0}, t_{0}\right) \in\left(0, x_{M}\right) \times \mathbb{R},
$$

for some $x_{M} \in(0,1)$. $V_{i n}$ and $V_{e}$ are defined as $V\left(\mathcal{T}_{\text {in }}\left(t_{0}, x, v\right) ; x, v, t_{0}\right)$ and $V\left(\mathcal{T}_{e}\left(t_{0}, x, v\right) ; x, v, t_{0}\right)$ respectively, where $\mathcal{T}_{\text {in }}\left(t_{0}, x, v\right)$ (resp. $\left.\mathcal{T}_{e}\left(t_{0}, x, v\right)\right)$ is $\sup \left\{t<t_{0}: X\left(t ; x, v, t_{0}\right)=0\right\}$ (resp. $\inf \{t>$ $\left.\left.t_{0}: X\left(t ; x, v, t_{0}\right)=0\right\}\right)$. The aim of the appendix is to prove that the potential $\Phi$ does not depend on time. First of all, we remark that for $x_{M}$ small enough, $\frac{\partial \Phi}{\partial x}>0$ on $\left(0, x_{M}\right)$ - see the first part of the proof of Theorem 2.5 - so that $V$ is positive on $\left(\mathcal{T}_{\text {in }}\left(t_{0}, x_{0}, 0\right), t_{0}\right)$ and negative on $\left(t_{0}, \mathcal{T}_{e}\left(t_{0}, x_{0}, 0\right)\right.$. Therefore, on each time interval, the position variable is a strictly monotone function of time $t$ that we shall denote by $X^{+}(t)$ and $X^{-}(t)$, for $t_{0} \in \mathbb{R}, x_{0} \in\left(0, x_{M}\right)$, fixed. The function $X^{+}$is a bijection from $\left(\mathcal{T}_{\text {in }}\left(t_{0}, x_{0}, 0\right), t_{0}\right)$ onto $\left(0, x_{0}\right)$ while $X^{-}$is a bijection from $\left(t_{0}, \mathcal{T}_{e}\left(t_{0}, x_{0}, 0\right)\right.$ onto $\left(x_{0}, 0\right)$. Letting $t^{+}$and $t^{-}$be their inverse functions, we have

$$
\frac{d t^{ \pm}}{d X}=\frac{1}{V}, \quad \frac{d V}{d X}=-\frac{1}{V} \frac{\partial \Phi}{\partial x}\left(X, t^{ \pm}\right)
$$

Let $e_{ \pm}(X)=\frac{1}{2} V^{2}(X)$. Then, simple algebraic computations lead to the following expressions:

$$
\begin{gathered}
t^{ \pm}(X)=t_{0} \mp \int_{x_{0}}^{X} \frac{d Y}{\sqrt{2 e_{ \pm}(Y)}} \quad \forall X \in\left[0, x_{0}\right], \\
\frac{d e_{ \pm}}{d X}=-\frac{\partial \Phi}{\partial x}\left(X, t^{ \pm}(X)\right), \quad e_{ \pm}\left(x_{0}\right)=0 .
\end{gathered}
$$

The condition $\left|V_{i n}\left(t_{0}, x_{0}, 0\right)\right|=\left|V_{e}\left(t_{0}, x_{0}, 0\right)\right|$ reads $e_{+}(0)=e_{-}(0)$. Next, we perform the rescaling:

$$
x_{0}=\varepsilon^{2}, \quad X=\varepsilon^{2}(1-x) \quad \text { and } \quad e_{ \pm}(X):=\frac{\varepsilon^{2}}{2} e_{ \pm}^{\varepsilon}(x)
$$

It is readily seen the $e_{ \pm}^{\varepsilon}$ satisfy the following integro-differential equations

$$
\frac{d e_{ \pm}^{\varepsilon}}{d x}=2 \frac{\partial \Phi}{\partial x}\left(\varepsilon^{2}(1-x), t_{0} \pm \varepsilon \int_{0}^{x} \frac{d y}{\sqrt{e_{ \pm}^{\varepsilon}(y)}}\right), \quad e_{ \pm}^{\varepsilon}(0)=0 .
$$

and the condition $\left|V_{i n}\left(t_{0}, x_{0}, 0\right)\right|=\left|V_{e}\left(t_{0}, x_{0}, 0\right)\right|$ is written

$$
e_{+}^{\varepsilon}(1)=e_{-}^{\varepsilon}(1)
$$

for any $\varepsilon>0$ small enough. The above equations can be shown to have unique solutions for $\varepsilon$ small enough. Moreover, these solutions have the following asymptotic expansion in $\varepsilon$ :

$$
e_{ \pm}^{\varepsilon}=\sum_{n=0}^{+\infty} \varepsilon^{n} e_{n}^{ \pm}
$$

The constraint $\left|V_{\text {in }}\left(t_{0}, x_{0}, 0\right)\right|=\left|V_{e}\left(t_{0}, x_{0}, 0\right)\right|$ yields $e_{n}^{+}(1)=e_{n}^{-}(1)$. 
Lemma A.1 With the above notations, for all $n \in \mathbb{I N}$, we have the following identities:

(i) $\frac{d e_{2 n}^{ \pm}}{d x}(x)=\frac{2(1-x)^{n}}{n !} \partial_{x}^{n+1} \Phi\left(0, t_{0}\right)$,

(ii) $\frac{d e_{2 n+1}^{ \pm}}{d x}(x)=\frac{ \pm 2(1-x)^{n}}{(n+1) !}\left(\int_{0}^{x} \frac{d y}{\sqrt{e_{0}(y)}}\right) \partial_{t} \partial_{x}^{n+1} \Phi\left(0, t_{0}\right)$,

(iii) $\partial_{t} \partial_{x}^{n+1} \Phi\left(0, t_{0}\right)=0$.

Proof. We first remark that (iii) is a direct consequence of (ii) and of the fact that $e_{2 n+1}^{+}(1)=e_{2 n+1}^{-}(1)$. In order to prove (i) and (ii), we insert the expansion of $e_{ \pm}^{\varepsilon}$ in (27) and identify the terms of the same power in $\varepsilon$. From the zeroth order term, we obtain $\frac{d}{d x} e_{0}^{ \pm}=2 \partial_{x} \Phi\left(0, t_{0}\right)$. The formulae for $e_{1}^{ \pm}$(i.e. (ii) with $n=0$ ) follow from the order 1 term. For the higher order terms, we proceed by induction. Namely, let $n \in \mathbb{N}$ be given and assume that (i), (ii) and (iii) hold up to the order $n$. Let us prove that they hold for $n+1$. Terms of order $\varepsilon^{2 n+2}$ in the right hand side of (27) are obtained by taking $n+1$ derivatives of $\partial_{x} \Phi$ with respect to $x, n$ derivatives with respect to $x$ and two with respect to $t, n-1$ derivatives with respect to $x$ and 4 with respect to $t, \cdots, 2 n+2$ with respect to $t$. Noticing that (iii) holds up to $n$ and for all times $t_{0} \in \mathbb{R}$, we deduce that the only non vanishing term in this expansion is the first one. This leads to (i) for the index $n+1$. In order to prove (ii), we proceed analogously. The only non vanishing term of order $2 n+3$ is the one corresponding to $n+1$ derivatives with respect to $x$ and one derivative with respect to $t$. All the other terms involve $t$ derivatives of $\partial_{x}^{k} \Phi(0, t)$ with $k \leq n$, and are therefore vanishing in view of (iii). This leads to (ii) (with $n$ replaced by $n+1)$.

A straightforward consequence is the following Corollary that we use in the proof of Theorem 2.5.

Corollary A.2 Let $\Phi:[0,1] \times \mathbb{R} \rightarrow \mathbb{R},(x, t) \mapsto \Phi(x, t)$ be an analytic function in $x$ with $C^{\infty}$ in time coefficients such that $\Phi(0, t)=0$ and $\Phi(1, t)=\Phi_{1}$ do not depend on $t$. Assume that $\frac{\partial \Phi}{\partial x}(x, t) \geq C_{2}>0$ uniformly in $(x, t) \in\left(0, x_{M}\right) \times \mathbb{R}$. If the characteristics $(X, V)(t ; x, v, s)$ defined by (26) are such that, with the above notations, $\left|V_{\text {in }}\left(t_{0}, x_{0}, 0\right)\right|=\left|V_{e}\left(t_{0}, x_{0}, 0\right)\right|$ for any $\left(x_{0}, t_{0}\right) \in\left(0, x_{M}\right) \times \mathbb{R}$, then $\Phi$ does not depend on $t$.

\section{B Two technical lemmata for the Bolza problem}

The Bolza problem is a standard question of mechanics. For a given potential $\psi$ and for any given pair $\left(x_{1}, x_{2}\right) \in \bar{\omega}^{2}$ of points, does there exist a trajectory which connects $x_{1}$ to $x_{2}$, for an appropriate initial velocity $v_{1}$ ? In this Appendix, we are going to prove two lemmata which are of interest for the proof of Corollary 5.3. We consider first the Bolza problem for two points $x_{1}, x_{2} \in \partial \omega$ such that the segment $\left(x_{1}, x_{2}\right)$ is contained in $\omega$, and then prove that two arbitrary points of the boundary of $\omega$ can be connected by a finite number of such segments, under the assumption that $\omega$ is a connected and bounded domain. For simplicity, we assume that $\psi$ is of class $C^{2}$, so that we deal with classical characteristics, but an extension based on the uniqueness of weaker notions of characteristics (see $[42,72])$ is easy to establish.

Let $x_{1}, x_{2} \in \partial \omega$. We shall say that

$$
\begin{gathered}
\left(x_{1}, x_{2}\right) \text { satisfy Property }(\mathcal{S}) \text { if and only if } \\
\nu\left(x_{i}\right) \cdot\left(x_{2}-x_{1}\right) \neq 0, i=1,2 \text {, and }\left(x_{1}, x_{2}\right)=\left\{t x_{1}+(1-t) x_{2}: t \in(0,1)\right\} \subset \omega .
\end{gathered}
$$

Let $u_{0}=\frac{x_{2}-x_{1}}{\left|x_{2}-x_{1}\right|}$. We may notice that if $(\mathcal{S})$ is satisfied, there exists an $\eta>0$ such that

$$
\forall u \in S^{d-1}, \quad\left|u-u_{0}\right|<\eta \quad \Longrightarrow \quad \begin{aligned}
& \left\{x_{1}+t u: t>0\right\} \cap \omega \\
& \text { is a neighborhood of }\left(x_{1}, x_{2}\right) \text { in } \omega .
\end{aligned}
$$


Lemma B.1 Let $\psi$ be a bounded $C^{2}$ potential defined on $\omega$ and consider $x_{1}, x_{2} \in \partial \omega$ such that $\left(x_{1}, x_{2}\right)$ has Property $(\mathcal{S})$. Then there exists an $A>0$ such that

$$
\forall a>A \quad \exists v_{1} \in a \cdot\left|S^{d-1}\right| \subset \mathbb{R}^{d},
$$

for which the characteristics defined in $\omega$ by

$$
\frac{d^{2} X}{d t^{2}}=-\nabla \psi(X), \quad X(0)=x_{1}, \quad \frac{d X}{d t}(0)=v_{1}
$$

ends at $x_{2}$.

Proof. For $\varepsilon>0$ and $u \in S^{d-1}$, we denote by $X_{\varepsilon, u}(t)$ the characteristics defined by

$$
\frac{d^{2} X_{\varepsilon, u}}{d t^{2}}=-\nabla \psi\left(X_{\varepsilon, u}\right), \quad X_{\varepsilon, u}(0)=x_{1}, \quad \frac{d X_{\varepsilon, u}}{d t}(0)=\frac{1}{\varepsilon} u
$$

in $\mathbb{R}^{d}$ (we extend $\psi$ by a bounded $C^{2}$ function to $\mathbb{R}^{d}$ ). Consider the time rescaling: $\varepsilon s=t$, and the rescaled characteristics $Y(\varepsilon, u, s)=X_{\varepsilon, u}\left(\frac{s}{\varepsilon}\right)$.

$$
\frac{\partial^{2} Y}{\partial s^{2}}=-\varepsilon^{2} \nabla \psi(Y), \quad Y(\varepsilon, u, 0)=x_{1}, \quad \frac{\partial Y}{\partial s}(\varepsilon, u, 0)=u .
$$

It is straightforward to prove that

$$
\left|\frac{\partial Y}{\partial s}(\varepsilon, u, s)-u\right| \leq \varepsilon \cdot \sqrt{2\|\psi\|_{L^{\infty}\left(\mathbb{R}^{d}\right)}} .
$$

Define $S(\varepsilon, u)=\inf \{s>0: Y(\varepsilon, u, s) \in \partial \omega\}$ and $Z(\varepsilon, u)=Y(\varepsilon, u, S(\varepsilon, u))$. By the above estimate, it is clear that, $Z\left(\varepsilon=0, u_{0}\right)=x_{2}$. The function $Z$ is of class $C^{2}$ on a neighborhood of $\left(0, u_{0}\right) \in \mathbb{R}^{+} \times S^{d-1}$, and it is easy to check that $\nabla_{u} Z\left(0, u_{0}\right)$ is invertible. The conclusion holds by the implicit functions theorem.

Lemma B.2 Let $x, y \in \partial \omega$. Assume that $\omega$ is a $C^{1}$ bounded and connected domain in $\mathbb{R}^{d}$ and $\psi$ is of class $C^{2}$ on $\omega$. Then there exists a finite sequence of points $x_{1}=x, x_{2}, \ldots x_{i}, x_{i+1}, \ldots x_{n-1}, x_{n}=y$ in $\partial \omega$ such that $\left(x_{i}, x_{i+1}\right)$ has Property $(\mathcal{S})$ for $i=1,2, \ldots n-1$.

Proof. We shall first prove an infinitesimal version of Lemma B.2. Let $x, y \in \partial \omega$ and denote by $\nu(x)$ and $\nu(y)$ the unit outgoing normals at $x$ and $y$ respectively. Because of the regularity of $\partial \omega$, for $\varepsilon>0$ small enough, if $|x-y|<\varepsilon$, there exists an $\eta>0$ such that

$$
\left\{z \in B(x, \varepsilon) \backslash\{x\}: \frac{z-x}{|z-x|} \cdot \nu(x)<-\eta\right\} \subset \omega \text { and } \quad\left\{z \in B(y, \varepsilon) \backslash\{y\}: \frac{z-y}{|z-y|} \cdot \nu(y)<-\eta\right\} \subset \omega .
$$

Next, consider

$$
\mathcal{U}=\left\{u \in S^{d-1}: u \cdot \nu(x)+\eta<0 \text { and } u \cdot \nu(y)+\eta<0\right\}
$$

for $\varepsilon>0$ small enough so that $\mathcal{U}$ is not empty (for $|x-y|<\varepsilon$ small enough, $|\nu(x)-\nu(y)|$ is as small as we want). Moreover, in the limit $\varepsilon \rightarrow 0$, we can take $\eta$ arbitrarily small. For any $u \in \mathcal{U}$, we may therefore consider

$$
\mathcal{Z}=\{z(u): u \in \mathcal{U}\}
$$

where $z(u)=x+t(x, u) u$ and $t(x, u)=\inf \left\{t>0: x+t u \in \omega^{c}\right.$ or $\left.(y, x+t u) \cap \omega^{c} \neq \emptyset\right\}$. By Sard's theorem, there exists at most a countable number of points $u$ in $\mathcal{U}$ for which either $(z(u)-x) \cdot \nu(z(u))=0$ or $(z(u)-x) \cdot \nu(z(u))=0$, which ends the proof: there exists a $u \in \mathcal{U}$ such that both $(x, z(u)) \subset \omega$ and $(z(u), y) \subset \omega$ have Property $\mathcal{S}$. By compactness of $\partial \omega$, if $x$ and $y$ are in the same connected component of $\partial \omega$, it is then easy to find a finite sequence of points $x_{1}=x, x_{2}, \ldots x_{i}, x_{i+1}, \ldots x_{n-1}$, $x_{n}=y$ in $\partial \omega$ with $\left|x_{i+1}-x_{i}\right|<\varepsilon$ for $\varepsilon>0$ small enough such that Lemma B.2 holds. If $x$ and $y$ are in two different connected components of $\partial \omega$, the extension is straightforward and left to the reader. $\square$ 


\section{Nonlinear stability}

We shall consider here the Vlasov-Poisson system in the whole space and see that (with no relaxation mechanism) the relative entropy is the same functional as the energy-Casimir functional which is used for studying the nonlinear stability of the solutions (see [49, 66, 9, 92, 93, 22] in the plasma physics case, $[61,60,58]$ for gravitational systems and for instance [59] for models with a magnetic field).

We will only sketch the main issues on stability at a formal level, to emphasize the connection with the relative entropy framework that we have used to study the irreversibility in the bounded domain case. For further details and stability in various norms, we refer to [22, 23].

Consider for simplicity the case $\omega=\mathbb{R}^{d}$ and let $f$ be a solution of the Vlasov-Poisson system in the presence of an external potential $\phi_{0}$ :

$$
\partial_{t} f+v \cdot \nabla_{x} f-\left(\nabla_{x} U[f]+\nabla_{x} \phi_{0}\right) \cdot \nabla_{v} f=0,
$$

where $U[f]=\left|S^{d-1}\right|^{-1}|x|^{-(d-2)} * \int_{\mathbb{R}^{d}} f d v$. Similar statements can be obtained in case of two species (and global electroneutrality) or for various boundary conditions (periodic, specular reflection, diffuse reflection,...). Note here that mass and energy are formally conserved and can a priori be fixed. Conditions on the growth of $\phi_{0}$ and on the decay of $\gamma$ have to be imposed to make the formulation of the problem consistent. These conditions will be referred as confinement conditions (see [46] for more details in the case $\left.\gamma(u)=e^{-u / \theta}\right)$. Assume that $\phi_{0} \in L_{\text {loc }}^{\infty}\left(\mathbb{R}^{d}\right)$ is such that $\lim _{|x| \rightarrow+\infty} \phi_{0}(x)=+\infty$ and $M_{0}(x, v)=\gamma\left(\frac{1}{2}|v|^{2}+\phi_{0}(x)\right)$ is bounded in $L^{1}\left(\mathbb{R}^{d}\right)$. Consider as in the case of a bounded domain $M$ verifying

$$
M(x, v)=\gamma\left(\frac{1}{2}|v|^{2}+\phi_{0}(x)+U[M](x)-\mu\right) .
$$

Such an $M$ can be defined by the mean of the convex functional:

$$
U \mapsto \frac{1}{2} \int_{\mathbb{R}^{d}}|\nabla U|^{2} d x-\int_{\mathbb{R}^{d}} G\left(U(x)+\phi_{0}(x)-\mu[U]\right) d x+M \mu[U],
$$

where $G(w)=2^{d / 2-1}\left|S^{d-1}\right| \cdot \int_{0}^{+\infty} s^{d / 2-1} \gamma(s+w) d s$ and $\mu=\mu[\phi]$ is fixed by the condition

$$
\int_{\mathbb{R}^{d}} \gamma\left(\frac{1}{2}|v|^{2}+\phi_{0}(x)+\phi(x)-\mu\right) d x=\mathcal{M}
$$

for an arbitrary given mass $\mathcal{M}>0$. We may notice that finding a solution $M$ is a well posed problem since $U[M]>0$ a.e. and $0 \leq M \leq M_{0}$ because $\gamma$ is decreasing (also see [47] for more details on the use of the decay properties of $\gamma$, for instance with respect to the existence of $\mu$ ). The total mass $\mathcal{M}$ is a monotone function of $\mu$ and is certainly well defined at least for $\mathcal{M}$ in some range.

Note now that provided $\|f(\cdot, \cdot, t)\|_{L^{1}\left(\mathbb{R}^{d} \times \mathbb{R}^{d}\right)}=\|M\|_{L^{1}\left(\mathbb{R}^{d} \times \mathbb{R}^{d}\right)}=\mathcal{M}$ and $f \geq 0$ a.e.,

$$
\begin{aligned}
\Sigma_{\gamma}[f \mid M] & =\int_{\mathbb{R}^{d} \times \mathbb{R}^{d}}\left(\beta_{\gamma}(f)+\frac{1}{2}|v|^{2}+\phi_{0}+\frac{1}{2} U[f]\right) d x d v-\int_{\mathbb{R}^{d} \times \mathbb{R}^{d}}\left(\beta_{\gamma}(M)+\frac{1}{2}|v|^{2}+\phi_{0}+\frac{1}{2} U[M]\right) d x d v \\
& =\int_{\mathbb{R}^{d} \times \mathbb{R}^{d}}\left(\beta_{\gamma}(f)-\beta_{\gamma}(M)-\beta_{\gamma}^{\prime}(M)(f-M)\right) d x d v+\frac{1}{2} \int_{\mathbb{R}^{d}}|\nabla U[f-M]|^{2} d x
\end{aligned}
$$

is formally conserved for any solution $f$. Since $\beta_{\gamma}^{\prime \prime}=-\left(\gamma^{-1}\right)^{\prime} \geq 0, \Sigma_{\gamma}[f \mid M]$ is actually a convex functional and certainly

$$
\Sigma_{\gamma}[f(\cdot, \cdot, t) \mid M] \leq \Sigma_{\gamma}\left[f_{0} \mid M\right]
$$

even for a weak solution. Minimizing

$$
\int_{\mathbb{R}^{d} \times \mathbb{R}^{d}}\left(\beta_{\gamma}(f)+\frac{1}{2}|v|^{2}+\phi_{0}(x)+\frac{1}{2} U[f]\right) d x d v
$$

under the constraint $\|f\|_{L^{1}\left(\mathbb{R}^{d} \times \mathbb{R}^{d}\right)}=\mathcal{M}$ is equivalent to find directly $M$ or to minimize (28). In this framework, $\mu$ is exactly the corresponding Lagrange multiplier (see [47] for a discussion for a 
model of nonlinear diffusion, which is completely similar to the kinetic case). As a consequence, $M$ and $\mu$ are uniquely defined. To conclude with these considerations on nonlinear stability, let us mention that $\Sigma_{\gamma}[f \mid M]$ is a measure of the distance between $f$ and $M$ : under the additional assumptions: $\inf _{(0,1)} \beta_{\gamma}^{\prime \prime}>0$ and $\inf _{t>0, h \in(0,1)} \beta_{\gamma}^{\prime \prime}(1+t h)(1+t)>0$ for instance, which are true if $\gamma^{-1}(f)=\mu-\theta \log \left(\frac{f}{1-\alpha f}\right)$ for $\theta>0, \alpha \geq 0$ and $\mu \in \mathbb{R}$, by the Csiszár-Kullback inequality (see for instance [6]), there exists a constant $C>0$ depending only on $\gamma$ such that

$$
\Sigma_{\gamma}[f(\cdot, \cdot, t) \mid M] \geq C\|f(\cdot, \cdot, t)-M\|_{L^{1}\left(\mathbb{R}^{d} \times \mathbb{R}^{d}\right)}^{2}+\frac{1}{2} \int_{\mathbb{R}^{d}}|\nabla U[f-M]|^{2} d x .
$$

The entropy functional $\Sigma_{\gamma}[f(\cdot, \cdot, t) \mid M]$ is therefore a measurement of the nonlinear stability of $M$ in the $L^{1}$ norm:

$$
\text { for any } \varepsilon>0 \text {, if } \Sigma_{\gamma}\left[f_{0} \mid M\right] \leq C \varepsilon^{2} \text {, then }\|f(\cdot, \cdot, t)-M\|_{L^{1}\left(\mathbb{R}^{d} \times \mathbb{R}^{d}\right)} \leq \varepsilon \text {. }
$$

Theorem 2.1 also provides a measurement of the nonlinear stability. Depending on the behaviour of $\beta_{\gamma}^{\prime \prime}$, this stability can be measured in various $L^{q}$ norms, with $q \in[1,2]$ (see [23]).

\section{Relation with nonlinear diffusion equations}

Throughout this paper, we have emphasized the analogies of the relative entropies for kinetic equations and nonlinear diffusions. In a simple case (corresponding again to $\omega=\mathbb{R}^{d}$ and for a specific collision kernel), we are going to make this connection precise by taking at a formal level an appropriate diffusive limit.

Consider a solution in $C^{0}\left(\mathbb{R}^{+}, L_{+}^{1}\left(\mathbb{R}^{d}\right)\right)$ of

$$
\rho_{t}=\Delta \nu(\rho)+\nabla\left(\rho \nabla \phi_{0}\right)+\varepsilon \nabla \cdot(\rho \nabla \phi)
$$

where either $\varepsilon=0$ (uncoupled case) or $\varepsilon=1$ and (Poisson coupling) $\phi=V[\rho]=\left|S^{d-1}\right|^{-1}|x|^{-(d-2)} * \rho$. Let $\Gamma$ be the inverse of $u \mapsto-\nu^{\prime}(u) / u$ and assume that it is a nonnegative decreasing function on $\mathbb{R}$. We denote by $\beta$ a primitive of $-\int_{0}^{u} \Gamma^{-1}(z) d z=\int_{0}^{u} \frac{\nu^{\prime}(z)}{z} d z$. The function $\rho_{\infty}(x)=\Gamma\left(\phi_{0}+\varepsilon V\left[\rho_{\infty}\right]-\mu\right)$ is a stationary solution of (29) and the convergence of $\rho(t, \cdot)$ to $\rho_{\infty}$ as $t \rightarrow+\infty$ is measured by

$$
\Sigma\left[\rho \mid \rho_{\infty}\right]=\int_{\mathbb{R}^{d}}\left(\beta(\rho)-\beta\left(\rho_{\infty}\right)-\beta^{\prime}\left(\rho_{\infty}\right)\left(\rho-\rho_{\infty}\right)\right) d x+\frac{\varepsilon}{2} \int_{\mathbb{R}^{d}}\left|\nabla V\left[\rho-\rho_{\infty}\right]\right|^{2} d x,
$$

which is nonnegative and decays according to

$$
\frac{d}{d t} \Sigma\left[\rho \mid \rho_{\infty}\right]=-\int_{\mathbb{R}^{d}} \rho\left|\nabla \phi_{0}+\nabla V[\rho]-\nabla\left(\Gamma^{-1}(\rho)\right)\right|^{2} d x=:-I[\rho] .
$$

Note that $I[\rho]=0$ if and only if $\rho=\rho_{\infty}$. By a Csiszár-Kullback inequality,, we get

$$
\Sigma\left[\rho \mid \rho_{\infty}\right] \geq C\left\|\rho-\rho_{\infty}\right\|_{L^{1}\left(R^{d}\right)}^{2}+\frac{\varepsilon}{2}\left\|\nabla U\left[\rho-\rho_{\infty}\right]\right\|_{L^{2}\left(R^{d}\right)}^{2} .
$$

These equations have been extensively studied recently and we shall quote $[7,34,35,29,85,27,28$, $69,16,36,37]$ for uncoupled cases corresponding to $(29)$ and $[5,15,18]$ for the coupled case. Also see $[24,47]$ for a study of the corresponding stationary solutions as well as the relation of $\Sigma\left[\rho \mid \rho_{\infty}\right]$ with the functional defined by (28).

The nonlinear diffusion equation (29) can be obtained from a singular perturbation of a kinetic equation. Namely, let $\gamma$ be a function which satisfies Property $\mathcal{P}$. For a given function $g(v)$, we define

$$
m_{g}(v)=\gamma\left(\frac{1}{2}|v|^{2}+a_{g}\right)
$$


where $a_{g}$ is the unique real number such that $\int_{\mathbb{R}^{d}} m_{g}(v) d v=\int_{\mathbb{R}^{d}} g(v) d v$. Consider the VlasovPoisson-Boltzmann system

$$
\partial_{t} f^{\eta}+\frac{1}{\eta}\left[v \cdot \nabla_{x} f^{\eta}-\left(\nabla_{x} \phi_{0}+\varepsilon \nabla_{x} \phi\right) \cdot \nabla_{v} f^{\eta}\right]=\frac{1}{\eta^{2}} Q\left(f^{\eta}\right)
$$

where $Q(g)=m_{g}-g$. With the notations of Sections 1-2, the operator $Q$ satisfies

$$
-D[g]=\int_{\mathbb{R}^{d}} Q(g)\left[\frac{1}{2}|v|^{2}-\gamma^{-1}(g)\right] d v=\int_{\mathbb{R}^{d}}\left(m_{g}-g\right)\left(\gamma^{-1}\left(m_{g}\right)-\gamma^{-1}(g)\right) d v \leq 0
$$

and the equality case holds if and only if $g=m_{g}$. The $H$-Theorem (see Theorem 2.1) takes the form

$$
\frac{d}{d t} \Sigma_{\gamma}\left[f^{\eta}(\cdot, \cdot, t) \mid M\right]=-\frac{1}{\eta^{2}} \int_{\mathbb{R}^{d}} D\left[f^{\eta}(x, \cdot, t)\right] d x
$$

We claim that the formal limit $f^{0}$ of $f^{\eta}$ is given by $f^{0}=\gamma\left(\frac{1}{2}|v|^{2}+a_{f_{0}}(x, t)\right)$ for some function $a_{f_{0}}(x, t)$ and that the corresponding density $\rho(x, t)=\int_{\mathbb{R}^{d}} f^{0}(x, v, t) d v$ satisfies (29) with $\nu(u)=$ $-\int_{0}^{u} s\left(\Gamma^{-1}\right)^{\prime}(s) d s$ and $\Gamma(u)=\int_{\mathbb{R}^{d}} \gamma\left(\frac{1}{2}|v|^{2}+u\right) d v$ (the functions $\rho$ and $a_{f^{0}}$ are linked to each other by the relation $\rho(x, t)=\Gamma\left(a_{f^{0}}(x, t)\right)$. To prove this claim, we first integrate the Boltzmann equation (30) with respect to $v$ and find

$$
\partial_{t} \rho^{\eta}+\operatorname{div}_{x} j^{\eta}=0 \quad \text { with } \rho^{\eta}=\int_{\mathbb{R}^{d}} f^{\eta} d v \text { and } j^{\eta}=\frac{1}{\eta} \int_{\mathbb{R}^{d}} v f^{\eta} d v .
$$

Then, multiplying (30) by $\eta v$ and integrating with respect to $v$, we find

$$
-j^{\eta}=\eta^{2} \partial_{t} j^{\eta}+\operatorname{div}_{x}\left[\int_{\mathbb{R}^{d}} v \otimes v f^{\eta} d v\right]+\left(\nabla \phi_{0}+\varepsilon \nabla \phi^{\eta}\right) \rho^{\eta}
$$

Passing to the limit $\eta \rightarrow 0$ formally yields

$$
\partial_{t} \rho+\operatorname{div}_{x} j=0 \quad \text { with }-j=\operatorname{div}_{x}\left[\int_{\mathbb{R}^{d}} v \otimes v \gamma\left(\frac{1}{2}|v|^{2}+a_{f^{0}}(x, t)\right) d v\right]+\left(\nabla \phi_{0}+\varepsilon \nabla \phi\right) \rho .
$$

We may notice that

$\operatorname{div}_{x}\left[\int_{\mathbb{R}^{d}}^{v} \otimes v \gamma\left(\frac{1}{2}|v|^{2}+a_{f^{0}}\right) d v\right]=\frac{1}{d} \nabla_{x} \int_{\mathbb{R}^{d}}|v|^{2} \gamma\left(\frac{1}{2}|v|^{2}+a_{f^{0}}\right) d v=-\left(\Gamma \circ a_{f^{0}}\right) \nabla_{x} a_{f^{0}}=\nabla_{x} \nu(\rho)$,

which leads to (29).

This formal limit holds not only at the level of the equations but also for the relative entropy. Consider the $H$-Theorem written for $f^{\eta}$ and let us carry out the limit $\eta \rightarrow 0$. A straightforward computation shows that $\Sigma_{\gamma}\left[f^{0} \mid M\right]$ is exactly equal to $\Sigma\left[\rho \mid \rho_{\infty}\right]$. Let us analyze the limit of $\frac{1}{\eta^{2}} \int_{\mathbb{R}^{d}} D\left[f^{\eta}\right] d x$ by the mean of a Hilbert expansion for $f^{\eta}$ : let

$$
f^{\eta}=f^{0}+\eta f_{1}+O\left(\eta^{2}\right)
$$

The equation satisfied by $f_{1}$ is

$$
D_{f^{0}} Q\left(f_{1}\right)=v \cdot \nabla_{x} f^{0}-\left(\nabla_{x} \phi_{0}+\varepsilon \nabla_{x} \phi\right) \cdot \nabla_{v} f^{0}=\gamma^{\prime}\left(\frac{1}{2}|v|^{2}+a_{f^{0}}\right) v \cdot\left(\nabla_{x} a_{f^{0}}-\nabla_{x} \phi_{0}-\varepsilon \nabla_{x} \phi\right)
$$

where $D_{f^{0}} Q$ is the linearization of $Q$ at $f=f^{0}$. A straightforward computation shows that

$$
D_{f^{0}} Q\left(f^{1}\right)=\gamma^{\prime}\left(\frac{1}{2}|v|^{2}+a\right)\left(\Gamma^{-1}\right)^{\prime}(\rho)\left(\int_{\mathbb{R}^{d}} f_{1} d v\right)-f_{1}
$$


Since $f_{1}$ is defined up to an element of the kernel of $D_{f^{0}} Q$, which is generated by the positive function $\gamma^{\prime}\left(\frac{1}{2}|v|^{2}+a\right)\left(\Gamma^{-1}\right)^{\prime}(\rho)$, we can choose $f^{1}$ such that $\int_{\mathbb{R}^{d}} f_{1} d v=0$. In this case, we have

$$
f_{1}=-D_{f^{0}} Q\left(f_{1}\right)=\gamma^{\prime}\left(\frac{1}{2}|v|^{2}+a\right) v \cdot\left(-\nabla_{x} a_{f^{0}}+\nabla_{x} \phi_{0}+\varepsilon \nabla_{x} \phi\right) .
$$

On the other hand

$$
\begin{aligned}
\lim _{\eta \rightarrow 0} \frac{1}{\eta^{2}} \int_{\mathbb{R}^{d}} D\left[f^{\eta}\right] d x & =\lim _{\eta \rightarrow 0}-\frac{1}{\eta^{2}} \int_{\mathbb{R}^{d}} Q\left(f^{\eta}\right)\left[\frac{1}{2}|v|^{2}-\gamma^{-1}\left(f^{\eta}\right)\right] d v d x \\
& =\int_{\mathbb{R}^{d}} D_{f^{0}} Q\left(f_{1}\right) \cdot\left(\gamma^{-1}\right)^{\prime}\left(f^{0}\right) f_{1} d v d x \\
& =-\int_{\mathbb{R}^{d}}\left(f_{1}\right)^{2}\left(\gamma^{-1}\right)^{\prime}\left(f^{0}\right) d v d x \\
& =-\int_{\mathbb{R}^{d}}\left\{\int_{\mathbb{R}^{d}} \gamma^{\prime}\left(\frac{1}{2}|v|^{2}+a\right)\left|\left(-\nabla_{x} a_{f^{0}}+\nabla_{x} \phi_{0}+\varepsilon \nabla_{x} \phi\right) \cdot v\right|^{2} d v\right\} d x \\
& =-\frac{1}{d} \int_{\mathbb{R}^{d}}\left\{\int_{\mathbb{R}^{d}}|v|^{2} \gamma^{\prime}\left(\frac{1}{2}|v|^{2}+a\right) d v\right\}\left|-\nabla_{x} a_{f^{0}}+\nabla_{x} \phi_{0}+\varepsilon \nabla_{x} \phi\right|^{2} d x \\
& =-\int_{\mathbb{R}^{d}} \rho\left|-\nabla_{x} a_{f^{0}}+\nabla_{x} \phi_{0}+\varepsilon \nabla_{x} \phi\right|^{2} d x,
\end{aligned}
$$

which is nothing else than $I(\rho)$ since $a_{f^{0}}=\Gamma^{-1}(\rho)$. This shows at a formal level that

$$
\Sigma_{\gamma}\left[f^{\eta}(\cdot, \cdot, t) \mid M\right]+\frac{1}{\eta^{2}} \int_{t}^{+\infty} d s \int_{\mathbb{R}^{d}} D\left[f^{\eta}(x, \cdot, s] d x\right.
$$

converges as $\eta \rightarrow 0$ to $\Sigma\left[\rho(\cdot, t) \mid \rho_{\infty}\right]+\int_{t}^{+\infty} d s \int_{\mathbb{R}^{d}} I[\rho(\cdot, s)] d x$.

Example. Consider the case of the Fermi-Dirac statistics. The kinetic equilibrium distribution function is the one which was exhibited in Examples 2 and 3 of Section 1 (also see [18, 75, 76, 47]): with the above notations, for any $(x, v) \in \mathbb{R}^{d} \times \mathbb{R}^{d}$,

$$
f_{\infty}(x, v)=\gamma\left(\frac{1}{2}|v|^{2}+\phi_{0}+\varepsilon \phi-\mu\right) \quad \text { with } \gamma(u)=\left(\alpha+e^{u}\right)^{-1}
$$

where $\alpha>0$ is a parameter related to Planck's constant. We refer to [55] for a mathematically rigourous justification of the diffusive limit. The function $\rho_{\infty}(x)=\Gamma\left(\phi_{0}+\varepsilon \phi-\mu\right)$ is the unique equilibrium density of the nonlinear diffusion equation

$$
\rho_{t}=\nabla \cdot(\nabla \nu(\rho)+\rho \nabla \phi)
$$

where $\nu(u)=-\int_{0}^{u} s\left(\Gamma^{-1}\right)^{\prime}(s) d s$ and $\Gamma(u)=\left|S^{d-1}\right| \int_{0}^{+\infty}(2 s)^{d / 2-1} \gamma(s+u) d s$.

Acknowledgments. The authors acknowledge support from the GdR SPARCH of the Centre National de la Recherche Scientifique (France) and from the T.M.R. network Asymptotic Methods in Applied kinetic Theory \# ERB FMRXCT97 0157, run by the European Community.

\section{References}

[1] R. Alexandre, Weak solutions of the Vlasov-Poisson initial-boundary value problem, Math. Meth. Appl. Sci. 16 no. 8 (1993), 587-607.

[2] L. Arkeryd, On the stationary Boltzmann equation in $R^{n}$, Internat. Math. Res. Notices 2000, no. $12,625-641$. 
[3] L. Arkeryd, A. Nouri, $L^{1}$ solutions to the stationary Boltzmann equation in a slab, Ann. Fac. Sci. Toulouse Math. (6) 9 no. 3 (2000), 375-413.

[4] L. Arkeryd, A. Nouri, The stationary Boltzmann equation in the slab with given weighted mass for hard and soft forces, Ann. Scuola Norm. Sup. Pisa 27 (1999), 533-566.

[5] A. Arnold, P. Markowich, G. Toscani, On large time asymptotics for drift-diffusion Poisson systems, Transport Theory Statist. Phys. 29 no. 3-5 (2000), 571-581.

[6] A. Arnold, P. Markowich, G. Toscani, A. Unterreiter, On generalized Csiszár-Kullback inequalities, Monatsh. Math. 131 no. 3 (2000), 235-253.

[7] A. Arnold, P. Markowich, G. Toscani, A. Unterreiter, On convex Sobolev inequalities and the rate of convergence to equilibrium for Fokker-Planck type equations, Comm. Partial Differential Equations 26 no. 1-2 (2001), 43-100.

[8] R. Balian, From microphysics to macrophysics, Vol. I. Methods and applications of statistical physics, Texts and Monographs in Physics, Springer-Verlag, Berlin, 1991.

[9] J. Batt, G. Rein, A rigorous stability result for the Vlasov-Poisson system in three dimensions. Ann. Mat. Pura Appl. (4) 164 (1993), 133-154.

[10] J. Batt, P.J. Morrison, and G. Rein, Linear stability of stationary solutions of the Vlasov-Poisson system in three dimensions, Arch. Rational Mech. Anal. 130 (1995), 163-182.

[11] N. Ben Abdallah, Weak solutions of the initial-boundary value problem for the Vlasov-Poisson System, Math. Meth. Appl. Sci. 17 (1994), 451-476.

[12] N. Ben Abdallah, J. Dolbeault, Entropies relatives pour le système de Vlasov-Poisson dans des domaines bornés [Relative entropies for the Vlasov-Poisson system in bounded domains], C. R. Acad. Sci. Paris Sér. I 320 (2000), 867-872.

[13] N. Ben Abdallah, L. Tayeb, Asymptotique de diffusion pour le système de Boltzmann-Poisson unidimensionnel [Diffusion approximation for the one-dimensional Boltzmann-Poisson system], C. R. Acad. Sci. Paris Sér. I Math. 329 no. 8 (1999), 735-740.

[14] S. Benachour, Analyticité des solutions des équations de Vlassov-Poisson, Ann. Scuola Norm. Sup. Pisa Cl. Sci. (4) 16 no. 1 (1989), 83-104.

[15] P. Biler, J. Dolbeault, Long time behavior of solutions to Nernst-Planck and Debye-Hückel drift-diffusion systems, Annales Henri Poincaré 1 no. 3 (2000), 461-472.

[16] P. Biler, J. Dolbeault, M.J. Esteban, Intermediate asymptotics in $L^{1}$ for general nonlinear diffusion equations, Preprint Ceremade no. 0031(2000), to appear in Appl. Math. Letters

[17] P. Biler, J. Dolbeault, M.-J. Esteban, G. Karch, Stationary solutions, intermediate asymptotics and large time behaviour of type II Streater's models, Adv. Diff. Eq. 6 no. 4 (2001), 461-480.

[18] P. Biler, J. Dolbeault, P. Markowich, Large time asymptotics of nonlinear drift-diffusion systems with Poisson coupling, Preprint Ceremade no. 9932 (1999), 1-16, to appear in Transport Th. Stat. Phys. 30 no. 4-6 (2001), 521-536.

[19] L.L. Bonilla, J.A. Carrillo, J. Soler, Asymptotic behaviour of the initial boundary value problem for the three-dimensional Vlasov-Poisson-Fokker-Planck system, SIAM J. Appl. Math. 57 (1997), $1343-1372$.

[20] F. Bouchut, Existence and uniqueness of a global smooth solution for the Vlasov-Poisson-FokkerPlanck system in three dimensions, J. Funct. Anal. 111 no. 1 (1993), 239-258. 
[21] F. Bouchut, J. Dolbeault, On long time asymptotics of the Vlasov-Fokker-Planck equation and of the Vlasov-Poisson-Fokker-Planck system with Coulombic and Newtonian potentials, Differential Integral Equations 8 no. 3 (1995), 487-514.

[22] P. Braasch, G. Rein, J. Vukadinović, Nonlinear stability of stationary plasmas - an extension of the energy-Casimir, SIAM J. Appl. Math. 59 no. 3 (1999), 831-844.

[23] M. J. Cáceres, J. A. Carrillo, J. Dolbeault Nonlinear Stability in $L^{p}$ for Solutions of the VlasovPoisson system for charged particles, Preprint Ceremade no. 0130 (2001), 1-18.

[24] L. Caffarelli, J. Dolbeault, P. Markowich, C. Schmeiser, On Maxwellian equilibria of insulated semiconductors, Interfaces and Free Boundaries 2 no. 3 (2000), 331-339.

[25] E. Caglioti, C. Maffei, Time asymptotics for solutions of Vlasov-Poisson equation in a circle, J. Statist. Phys. 92 no. 1-2 (1998), 301-323.

[26] J.A. Carrillo, Global weak solutions for the initial-boundary value problems to the VlasovPoisson-Fokker-Planck system, Math. Meth. Appl. Sci. 21 (1998), 907-938.

[27] J.A. Carrillo, A. Jüngel, P. Markowich, G. Toscani, A. Unterreiter, Entropy dissipation methods for degenerate parabolic problems and generalized Sobolev inequalities, Monatsh. Math. 133 no. 1 (2001), 1-82.

[28] J.A. Carrilo, P.A. Markowich, A. Unterreiter, Large-time asymptotics of porous-medium type equations, GAKUTO Internat. Ser. Math. Sci. Appl. 13 (2000), 24-36.

[29] J.A. Carrillo, G. Toscani, Asymptotic $L^{1}$-decay of solutions of the porous medium equation to self-similarity, Indiana Univ. Math. J. 49 (2000), 113-141.

[30] C. Cercignani, R. Illner, M. Pulvirenti, The mathematical theory of dilute gases, Applied Mathematical Sciences 106, Springer-Verlag, New York, 1994.

[31] J. Darrozès, J.-P. Guiraud, Généralisation formelle du théorème $H$ en présence de parois. Applications, C. R. Acad. Sci. Paris Sér. A 262 (1966), 1368-1371.

[32] P. Degond, Spectral theory of the linearized Vlasov-Poisson equation, Trans. Amer. Math. Soc. 294 no. 2 (1986), 435-453.

[33] P. Degond, T. Goudon, F. Poupaud, Diffusion limit for nonhomogeneous and non-microreversible processes, Indiana Univ. Math. J. 49 no. 3 (2000), 1175-1198.

[34] M. Del Pino, J. Dolbeault, Generalized Sobolev inequalities and asymptotic behaviour in fast diffusion and porous medium problems, Preprint Ceremade no. 9905 (1999), 1-45 \& Preprint TMR "Asymptotic Methods in Kinetic Theory" no. 50 (1999), 1-45.

[35] M. Del Pino \& J. Dolbeault, Best constants for Gagliardo-Nirenberg inequalities and application to nonlinear diffusions, Preprint Ceremade no. 0119 (2001), 1-25, to appear in J. Math. Pures Appl.

[36] M. Del Pino \& J. Dolbeault, Non linear diffusions and optimal constants in Sobolev type inequalities: asymptotic behaviour of equations involving the $p$-Laplacian, Preprint Ceremade no. 0126 (2001), 1-6.

[37] M. Del Pino \& J. Dolbeault, Asymptotic behaviour of nonlinear diffusions, Preprint Ceremade no. 0127 (2001), 1-8.

[38] L. Desvillettes, J. Dolbeault, On long time asymptotics of the Vlasov-Poisson-Boltzmann equation, Comm. Partial Differential Equations 16 no. 2-3 (1991), 451-489. 
[39] L. Desvillettes, C. Villani, On the trend to golbal equilibrium in spatially inhomogeneous entropy-dissipating systems, Part I: the linear Fokker-Planck equation, Comm. Pure Appl. Math. 54 no. 1 (2001), 1-42.

[40] L. Desvillettes, C. Villani, On the trend to global equilibrium in spatially inhomogeneous entropy-dissipating systems, Part II. H-theorem and applications, Comm. Partial Differential Equations 25 no. 1-2 (2000), 261-298.

[41] R.-J. DiPerna, P.-L. Lions, Solutions globales d'équations du type Vlasov-Poisson, C. R. Acad. Sci. Paris 307 (1988), 655-658.

[42] R.-J. DiPerna, P.-L. Lions, Ordinary differential equations, transport theory and Sobolev spaces, Invent. Math. 98 no. 3 (1989), 511-547.

[43] R.-J. DiPerna, P.-L. Lions, On the Cauchy problem for Boltzmann equations: global existence and weak stability, Ann. of Math. 130 no. 2 (1989), 321-366.

[44] R.-J. DiPerna, P.-L. Lions, Global solutions of Boltzmann's equation and the entropy inequality, Arch. Rational Mech. Anal. 114 no. 1 (1991), 47-55.

[45] J. Dolbeault, Kinetic models and quantum effects: a modified Boltzmann equation for FermiDirac particles, Arch. Rational Mech. Anal. 127 no. 2 (1994), 101-131.

[46] J. Dolbeault, Free energy and solutions of the Vlasov-Poisson-Fokker-Planck system: external potential and confinement (large time behavior and steady states), J. Math. Pures Appl. 78 (1999), 121-157.

[47] J. Dolbeault, P. Markowich, A. Unterreiter, On Singular Limits of Mean-Field Equations, Arch. Rational Mech. Anal. 158 no. 4 (2001), 319-351.

[48] J. Dolbeault, G. Rein, Time-dependent rescalings and Lyapunov functionals for the VlasovPoisson and Euler-Poisson systems, and for related models of kinetic equations, fluid dynamics and quantum physics, Math. Models Methods Appl. Sci. 11 no. 3 (2001), 407-432.

[49] C.S. Gardner, Bound on the energy available from a plasma, Phys. Fluids 6 (1963), 839-840.

[50] P. Gérard, Solutions globales du problème de Cauchy pour l'équation de Boltzmann (d'après R.-J. Di Perna et P.-L. Lions), Sém. Bourbaki (40ème année) 699 (juin 1988).

[51] R. Glassey, J. Schaeffer, On time decay rates in Landau damping, Comm. Partial Differential Equations 20 no. 3-4 (1995), 647-676.

[52] F. Golse, From kinetic to macroscopic models, Kinetic equations and asymptotic theory, (B. Perthame \& L. Desvillettes eds.), Series in Applied Mathematics, Gauthier-Villars/ Elsevier, 2000.

[53] F. Golse, P.-L. Lions, B. Perthame and R. Sentis, Regularity of moments of the solution of a transport equation, J. Func. Anal. 76 (1988), 110-125.

[54] F. Golse, B. Perthame and R. Sentis, Un résultat de compacité pour les équations de transport et application au calcul de la limite de la valeur propre principale d'un opérateur de transport, C. R. Acad. Sci. Paris Série I 301 (1985), 341-344.

[55] F. Golse, F. Poupaud, Limite fluide des équations de Boltzmann des semi-conducteurs pour une statistique de Fermi-Dirac [Fluid limit of Boltzmann semiconductor equations for a Fermi-Dirac statistic], Asymptotic Anal. 6 no. 2 (1992), 135-160. 
[56] C. Greengard, P.A. Raviart, A boundary-value problem for the stationary Vlasov-Poisson equations: the plane diode, Comm. Pure Appl. Math. 43 no. 4 (1990), 473-507.

[57] Y. Guo, Stable magnetic equilibria in collisionless plasmas, Comm. Pure Appl. Math. 50 (1997), 891-933.

[58] Y. Guo, Variational method for stable polytropic galaxies, Arch. Rational Mech. Anal. 150 no. 3 (1999), 209-224.

[59] Y. Guo, Stable magnetic equilibria in a symmetric collisionless plasma, Comm. Math. Phys. 200 no. 1 (1999), 211-247.

[60] Y. Guo, G. Rein, Existence and stability of Camm type steady states in galactic dynamics, Indiana Univ. Math. J. 48 no. 4 (1999), 1237-1255.

[61] Y. Guo, G. Rein, Stable steady states in stellar dynamics, Arch. Rational Mech. Anal. 147 (1999), no. 3, 225-243.

[62] Y. Guo, W.A. Strauss, Instability of periodic BGK equilibria, Comm. Pure Appl. Math. 48 (1995), no. 8, 861-894.

[63] Y. Guo, W.A. Strauss, Unstable BGK solitary waves and collisionless shocks, Comm. Math. Phys. 195 (1998), no. 2, 267-293.

[64] Y. Guo, W.A. Strauss, Unstable oscillatory-tail waves in collisionless plasmas, SIAM J. Math. Anal. 30 (1999), no. 5, 1076-1114.

[65] Y. Guo, W.A. Strauss, Magnetically created instability in a collisionless plasma, J. Math. Pures Appl. (9) 79 (2000), no. 10,975-1009.

[66] D. Holm, J.E. Marsden, E. Jerrold, T. Raţiu, A. Weinstein, Nonlinear stability of fluid and plasma equilibria, Phys. Rep. 123 no. 1-2 (1985).

[67] E. Hörst, and R. Hunze, Weak solutions of the initial value problem for the unmodified nonlinear Vlasov equation, Math. Methods Appl. Sc. 6 (1984), 262-279.

[68] R. Illner, G. Rein, Time decay of the solutions of the Vlasov-Poisson system in the plasma physical case, Math. Methods Appl. Sci. 19 no. 17 (1996), 1409-1413.

[69] A. Jüngel, P. Markowich, G. Toscani, Decay rates for degenerate parabolic equations, USA-Chile Workshop on Nonlinear Analysis, Electron. J. Diff. Eqns., Conf. 6 (2001), 189-202.

[70] M. Lemou, M. Linearized quantum and relativistic Fokker-Planck-Landau equations, Math. Methods Appl. Sci. 23 no. 12, (2000), 1093-1119.

[71] Z. Lin, Instability of periodic BGK waves, Preprint (2001).

[72] P.-L. Lions, Sur les équations différentielles ordinaires et les équations de transport, C. R. Acad. Sci. Paris Sér. I 326 (1998), 833-838.

[73] P.-L. Lions, and B. Perthame, Propagation of moments and regularity for the Vlasov-Poisson system, Invent. Math., (105 (1991), 415-430.

[74] P. A. Markowich, F. Poupaud, C. Schmeiser, Diffusion approximation of nonlinear electron phonon collision mechanisms, RAIRO Modél. Math. Anal. Numér. 29 no. 7 (1995), 857-869.

[75] P.A. Markowich, C. Ringhofer, C. Schmeiser, Semiconductor Equations, Springer-Verlag, Wien, 1990. 
[76] P.A. Markowich, A. Unterreiter, Vacuum solutions of a stationary drift-diffusion model, Ann. Sc. Norm. Sup. Pisa 20 (1993), 371-386.

[77] S. Mischler, Uniqueness for the BGK equation in the all space and rate of convergence for a semi-discrete scheme, Differential and Integral Equations 9 no. 5 (1996), 111-1138.

[78] S. Mischler, On the trace problem for the solutions of the Vlasov equation, Comm. Partial Differential Equations 25 no. 7-8 (2000), 1415-1443.

[79] S. Mischler, On the initial boundary value problem for the Vlasov-Poisson-Boltzmann system, Comm. Math. Phys. 210 no. 2 (2000), 447-466.

[80] S. Mischler, On weak-weak convergences and application to the initial boundary value problem for kinetic equations, Preprint no. 35 of the University of Versailles.

[81] F.-J. Mustieles, Global existence of solutions of the nonlinear Boltzmann equation of semiconductor physics, Rev. Mat. Iberoamericana 6 no. 1-2 (1990), 43-59.

[82] F.-J. Mustieles, Global existence of weak solutions for a system of nonlinear Boltzmann equations in semiconductor physics, Math. Methods Appl. Sci. 14 no. 2 (1991), 139-153.

[83] J. Nieto, F. Poupaud, J. Soler, High-field limit for the Vlasov-Poisson-Fokker-Planck system, Arch. Rational Mech. Anal. 158 no. 1 (2001), 29-59.

[84] A. Nouri, F. Poupaud, Boundary value problem of the Vlasov-Boltzmann-Maxwell system under Fermi-Dirac statistics, SIAM J. Math. Anal. 26 no. 5 (1995), 1143-1156.

[85] F. Otto, The geometry of dissipative evolution equations: the porous medium equation, Comm. Partial Differential Equations 26 no. 1-2 (2001), 101-174.

[86] B. Perthame, Time decay, propagation of low moments and dispersive effects for kinetic equations, Comm. Partial Differential Equations 21, no. 3-4 (1996, 659-686.

[87] F. Poupaud, On a system of non linear Boltzmann equations of semiconductor physics, SIAM J. Appl. Math. 50 no. 6 (1990), 1593-1606.

[88] F. Poupaud, Solutions stationnaires des équations de Vlasov-Poisson, C. R. Acad. Sci. Paris, Série I 311 (1990), 307-312.

[89] F. Poupaud, Boundary value problems for the stationary Vlasov-Maxwell system, Forum Math. 4 (1992), 499-527.

[90] F. Poupaud, J. Soler, Boundary value problems for the stationary Vlasov-Maxwell system, Forum Math. 4 (1992), 499-527.

[91] F. Poupaud, J. Soler, Parabolic limit and stability of the Vlasov-Fokker-Planck system, Math. Models Methods Appl. Sci. 10 no. 7 (2000), 1027-1045.

[92] G. Rein, Non-linear stability for the Vlasov-Poisson system - the energy-Casimir method, Math. Meth. Appl. Sci. 17 no. 14 (1994), 1129-1140.

[93] J. Weckler, On the initial-boundary-value problem for the Vlasov-Poisson system: existence of weak solutions and stability, Arch. Rational Mech. Anal. 130 (1995), 145-161.

[94] G. Wolansky, On nonlinear stability of polytropic galaxies, Ann. Inst. H. Poincaré Anal. Non Linéaire 16 (1999), 15-48.

[95] P.E. Zhidkov, On a problem of two-time data for the Vlasov equation, Nonlinear Analysis T.M.A. 31 no. 5-6 (1998), 537-547. 